\title{
Classification of oral bioavailability of drugs by machine learning approaches: a comparative study
}

\author{
Rajnish Kumar ${ }^{1,2}$, Anju Sharma ${ }^{1,2}$, Pritish Varadwaj ${ }^{1}$, Ausaf Ahmad² and Ghulam Md Ashraf ${ }^{2}$ \\ Manuscript received on March 9, 2011 / accepted on August 15, 2011
}

\begin{abstract}
Oral Bioavailability is the rate and extent to which an active drug substance is absorbed and becomes available to the general circulation. A computational model for the prediction of oral bioavailability is a vital initial step in the drug discovery. It is decisive for selecting the promising compounds for the next level optimizations and recognition for the clinical trials. In the present investigation we aimed to perform the oral bioavailability prediction by comparing three machine learning methods i.e. Support Vector Machine (SVM) based kernel learning, Artificial Neural Network (ANN) and Bayesian classification approach. The overall prediction efficiency of SVM based model for the test set was $96.85 \%$, whereas according to the Bayesian classifier and ANN methods prediction efficiency was found to be $92.19 \%$ and $94.53 \%$ respectively. Thus the present results clearly suggested that the SVM based prediction of oral bioavailability of drugs is more efficient binary classification approach for the data under consideration.
\end{abstract}

Keywords: Artificial Neural Network, Bayesian classification, oral bioavailability, prediction, Support Vector Machine.

\section{INTRODUCTION}

Bioavailability is the amount of drug that reaches to the blood in an unchanged form, to carry out its pharmacological and therapeutic effect. It confers the rate at which the drugs get absorbed and the total amount that reaches to the systemic circulation. Understanding the bioavailability of a concern drug is crucial as the drug has to undergo a number of complicated biochemical pathways before producing the desired therapeutic effect. Hence, high oral bioavailability is among the most important consideration during drug development process.

There exists a plethora of studies to predict oral bioavailability which indicates that it is incredibly rich area of research. Oral bioavailability is usually determined in the preclinical stage of drug development process. Therefore, there is a need of a robust and accurate computational model which can predict the oral bioavailability of compounds without carrying out experiments. Various attempts in estimating oral bioavailability are reported in literature belonging to different categories viz. statistical anaIysis on known oral bioavailable drugs [12, 17], mechanistic models [20, 22], QSAR/QSPR models [16, 10], genetic programming [3, 9], Artificial Neural Networks (ANN), machine learning classification etc [19, 13].

Classification is a process of developing a function which describes and/or distinguishes data on the bases of attributes [13]. Classification is a two step process. First is the learning or training step. It involves development of classifier describing the predetermined set of classes using a training set. This is also called supervised learning as classifier learns from a dataset in which classes are defined for each data. Second step involves testing of the classifier developed, in which a new data independent of the training set is used to determine the accuracy of clas-

Correspondence to: Anju Sharma - E-mail: anjusharma.online@gmail.com

${ }^{1}$ Department of Bioinformatics, Indian Institute of Information Technology Allahabad, Deoghat, Jhalwa, Allahabad-211012, Uttar Pradesh, India.

${ }^{2}$ Amity Institute of Biotechnology (AIB), Amity University Uttar Pradesh (AUUP), Lucknow-226010, Uttar Pradesh, India. 
sifier. The function thus developed is used to predict class of data whose class label is unknown. Data can be classified using different approaches like ANN, Bayesian classifiers, Support Vector Machine (SVM) etc.

\section{MATERIALS AND METHODS}

\subsection{Data collection and data pre-processing}

\subsubsection{Collection of drugs}

The oral-bioavailability dataset used in this study was obtained from seven different sources [21, 6, 25, 15, 4, 8, 11]. Each drug molecule was represented with their chemical formulae and their bioavailability values. Dataset for this study comprises of total 511 drugs which are chemically diverse. Further the dataset of 511 drug molecules are randomly split into training and test set using 3:1 principle. Training set consists of 384 drugs whereas the test set consists of 127 drugs.

\subsubsection{Calculation of descriptor values}

Initially total 29 descriptors (physicochemical properties) were collected from various sources. The two softwares used for the calculation of all descriptors were the commercial versions of DRAGON [18] and TSAR (Supplementary: Tables 1-2).

In our models we used diverse data set in order to avoid any biasness in it. For this purpose values of descriptors were plotted against each other to examine the diversity among the descriptors (Fig. 1).

\subsubsection{Selection of optimal descriptors}

In order to minimize the dimension feature space (a possible outcome of large number of descriptors), we have selected Sequential Forward Feature Selection (SFFS) algorithm. This facilitates identifying and removing the irrelevant and redundant information as much as possible to improve the performance of machine learning algorithms.

SFFS algorithm starts with an empty set of features. In first iteration, algorithm considers all feature subsets with only one feature. Feature subset with higher accuracy is used as basis of next iteration. Iteratively, algorithm tentatively adds to the basis each feature which was not previously selected and retains the feature subset that results in the highest estimated performance. The search terminates after the accuracy of the current subset cannot be improved by adding any other feature [1]. SFFS is stated as: Given a feature set $X=\{x i \mid i=1 \ldots N\}$, find a subset $Y M=\{x i \ldots x M\}$, with $M<N$, which optimizes an objective function $J(Y)$. SFFS algorithm can be seen in Appendix.

Thus by implementing the SFFS algorithm we identified 12 descriptors as optimal features and selected for generation of the prediction model. These descriptors include Molecular Mass (MA), Total Hydrogen Count $(\mathrm{HC})$, Total Polar Surface Area (TPSA), Partition Coefficient (logP), Rotatable Bonds (RTB), Shape Flexibility Index (SFI), Molecular Volume, Molecular Refractivity [14], Molecular Surface Area (MSA), Solubility index (logS) [23], Count of Hydroxyl groups (HYG) [22] and Sum of E- states indices (SESI) [5].

\subsection{Implementation of classification approaches}

In present study, the aim is to construct a classifier for a given drug dataset which can differentiate drugs into two classes on the basis of their physicochemical properties. Classification is performed in such a way that each drug in the training data corresponds to a particular class and is represented by a set of features. Before employing any classification approach, assignment of class labels to whole drug data set was required. The constructed classifier is applied on the independent drug data set in order to predict the class label for it. We have defined two classes for the dataset 'low orally bioavailable (LO, less than 30 percent)' and 'high orally bioavailable ( $\mathrm{HO}$, greater than or equal to 30 percent)' [6]. Class labels were defined as 0 and 1 for $\mathrm{LO}$ and $\mathrm{HO}$ respectively.

In order to select suitable classifier, it was crucial to find out whether the data is linear or non-linear. We plotted the two defined classes for each of 12 descriptors. The distributions of data points for both the classes were found to be diverse, (Fig. 2) so non-linear classifiers were selected. In this study Support Vector Machine (SVM), Artificial Neural Network (ANN) and Bayesian classifiers were implemented to classify the drugs into $\mathrm{LO}$ and $\mathrm{HO}$.

\subsubsection{Classification by Support Vector Machine}

In the present study LibSVM package (version 2.81) [2] was used to implement SVM. The training dataset was used as input for SVM based classification and following steps were performed:

a) Scaling of data: Descriptors values were scaled within a numeric range -1 and 1 [7].

b) Kernel Selection: In LibSVM, there are four kernels [linear, sigmoid, gaussian, radial basis function (RBF)]. Individually all kernels were tried to determine best kernel in terms of accuracy. As we observed the best efficiency in RBF, so it was used for classification in the current study.

c) Determination of optimal parameters [cost factor, $(C)$ and kernel parameter $(\gamma)$ ]: Grid search was used to determine optimal value of $C$ and $\gamma$, which was found to be 512 and 0.0078125 respectively. Finally the classification model was generated using the best parameters $C$ and $\gamma$. 
RAJNISH KUMARA, ANJU SHARMA, PRITISH VARADWAJ, AUSAF AHMAD and GHULAM MD ASHRAF

Table 1 - Supplementary data set consisting 511 drugs with respective values of all 12 descriptors under study.

\begin{tabular}{|c|c|c|c|c|c|c|c|c|c|c|c|c|}
\hline Name of drug & $\begin{array}{l}\text { Molecular } \\
\text { mass }\end{array}$ & $\begin{array}{c}\text { Molecular } \\
\text { surface } \\
\text { area }\end{array}$ & $\begin{array}{l}\text { Molecular } \\
\text { volume }\end{array}$ & $\begin{array}{l}\text { Molecular } \\
\text { refractivity }\end{array}$ & \begin{tabular}{|c|} 
Total $\mathrm{H}$ \\
bond \\
counting
\end{tabular} & $\log P$ & $\begin{array}{l}\text { Rotable } \\
\text { bonds }\end{array}$ & TPSA & $\log S$ & $\begin{array}{c}\text { Shape } \\
\text { flexibility } \\
\text { index }\end{array}$ & \begin{tabular}{|c|} 
Sum of \\
E-states \\
indices \\
\end{tabular} & $\begin{array}{l}\text { Hydroxyl } \\
\text { groups }\end{array}$ \\
\hline 5-fluorouracil & 128.07 & 79.7582 & 44.7132 & 26.1802 & 4 & -0.0095 & 0 & 65.724 & -0.43 & 1.69653 & 35 & 0 \\
\hline AAFC(flurocitabine) & 243.22 & 145.887 & 91.6692 & 51.9663 & 8 & -0.2407 & 1 & 100.602 & -1.38 & 2.79835 & 50.8333 & 2 \\
\hline Abacavir & 286.38 & 183.954 & 103.261 & 80.8409 & 7 & 0.5408 & 4 & 101.888 & -2.37 & 3.78783 & 46.1667 & 1 \\
\hline acarbose & 645.691 & 298.801 & 230.876 & 137.602 & 33 & -4.8887 & 9 & 321.161 & -0.64 & 12.0643 & 133.5 & 13 \\
\hline acebutolol & 336.48 & 189.233 & 132.848 & 93.0872 & 8 & 0.976599 & 10 & 87.658 & -3.29 & 8.77703 & 59.5 & 1 \\
\hline Acetaminophen & 151.18 & 125.935 & 67.6326 & 40.8343 & 4 & 0.6111 & 1 & 49.326 & -1.56 & 2.35543 & 30.5 & 1 \\
\hline Acetylcysteine & 66.397 & 163.21 & 167.636 & 37.6118 & 6 & -0.9059 & 3 & 119.425 & -1.51 & 4.25662 & 31.8889 & 1 \\
\hline Acetylsalicylicacid & 180.17 & 126.225 & 69.8522 & 43.9488 & 5 & 1.2439 & 3 & 63.604 & -2.09 & 2.8925 & 40.1667 & 1 \\
\hline Acrivastine & 348.48 & 212.963 & 135.437 & 105.979 & 5 & 4.222 & 8 & 53.429 & -4.54 & 6.42962 & 57.5 & 1 \\
\hline Acyclovir & 225.24 & 158.205 & 87.7105 & 52.5548 & 8 & -1.1145 & 4 & 119.065 & -1.44 & 3.78369 & 44.1667 & 1 \\
\hline adefovir & 273.22 & 253.531 & 176.349 & 62.3878 & 10 & -0.1804 & 5 & 136.394 & -2.13 & 4.7622 & 51.3056 & 2 \\
\hline Albuterol & 324.43 & 197.273 & 112.472 & 82.7714 & 6 & 1.4333 & 4 & 92.338 & -3.83 & 5.44085 & 58.8167 & 0 \\
\hline Alfentanil & 85.504 & 416.59 & 392.895 & 118.591 & 6 & 2.4672 & 9 & 324.532 & -3.15 & 8.48888 & 67.25 & 0 \\
\hline Allopurinol & 136.13 & 102.837 & 52.9583 & 34.7296 & 6 & 0.3567 & 0 & 74.695 & -0.79 & 1.63289 & 26.5 & 1 \\
\hline $\begin{array}{l}\text { Alpha-acetyl-methadol(laam) } \\
\text { alpha-acetyl-methadol(laam)- }\end{array}$ & 353.55 & 207.436 & 114.961 & 107.362 & 3 & 4.9838 & 9 & 29.543 & -5.3 & 7.12671 & 54.4167 & 0 \\
\hline levacetylmethadol & 353.55 & 189.416 & 128.183 & 107.362 & 3 & 4.9838 & 9 & 29.543 & -5.3 & 7.12671 & 54.4167 & 0 \\
\hline alpha-Lipoic_acid & 7.3 & 206.34 & 211.385 & 54.2876 & 3 & 1.7823 & 5 & 154.623 & -2.96 & 5.10803 & 26.2667 & 1 \\
\hline alprazolam & 308.79 & 158.159 & 101.068 & 87.9249 & 3 & 4.3332 & 1 & 43.082 & -3.98 & 3.84379 & 43.4778 & 0 \\
\hline Alprenolol & 249.39 & 161.078 & 105.644 & 74.6616 & 5 & 2.8394 & 8 & 41.489 & -3.12 & 6.64849 & 39.5 & 1 \\
\hline Alprostadil & 94.83 & 354.54 & 402.82 & 98.3179 & 8 & 3.9292 & 14 & 292.455 & -3.65 & 10.856 & 63.1667 & 3 \\
\hline Amantadine & 151.28 & 116.729 & 58.9131 & 45.5344 & 2 & 1.1063 & 0 & 26.023 & -3.25 & 1.07107 & 18.25 & 0 \\
\hline Amdinocillin & 73.21 & 325.47 & 314.728 & 84.1107 & 5 & 1.5823 & 4 & 245.375 & -2.52 & 4.69977 & 51.2167 & 1 \\
\hline Amikacin & 331.95 & 585.7 & 492.805 & 129.841 & 30 & -6.9063 & 10 & 419.529 & -1.07 & 12.0936 & 118 & 8 \\
\hline Amiloride & 229.66 & 153.019 & 80.1756 & 56.2685 & 8 & -0.5551 & 2 & 156.803 & -2.57 & 4.43618 & 43.8111 & 0 \\
\hline aminopyrine & 231.33 & 154.019 & 90.378 & 70.508 & 1 & 1.428 & 2 & 30.177 & -0.7 & 3.10667 & 37.6667 & 0 \\
\hline Amiodarone & 645.35 & 266.784 & 165.212 & 143.144 & 4 & 7.4205 & 11 & 42.683 & -5.13 & 9.5127 & 61.5487 & 0 \\
\hline Amitriptyline & 277.44 & 194.395 & 108.252 & 92.3311 & 1 & 4.5186 & 4 & 3.238 & -4.79 & 4.21179 & 38.3333 & 0 \\
\hline Amlodipine & 408.92 & 229.026 & 132.042 & 108.637 & 8 & -0.1487 & 10 & 99.894 & -4.74 & 8.49814 & 66.9778 & 0 \\
\hline Amoxicillin & 365.44 & 198.152 & 123.741 & 89.123 & 10 & -0.1493 & 4 & 132.957 & -2.58 & 4.56231 & 69.05 & 2 \\
\hline Amphotericin B & 924.211 & 884.51 & 247.282 & 244.668 & 30 & 2.4159 & 3 & 319.609 & -4.05 & 23.8939 & 174.583 & 11 \\
\hline Ampicillin & 349.44 & 173.334 & 98.7257 & 87.4289 & 8 & 0.135099 & 4 & 112.729 & -2.76 & 4.34899 & 63.3833 & 1 \\
\hline amrinone & 348.9 & 174.452 & 121.638 & 95.5559 & 4 & 2.056 & 1 & 54.37 & -4.63 & 3.63865 & 52.8111 & 1 \\
\hline Amrinone-inamrinone & 187.22 & 146.613 & 75.4471 & 55.0668 & 4 & -0.8474 & 1 & 71.777 & -1.15 & 2.48019 & 35.1667 & 0 \\
\hline anastrozole & 293.41 & 162.233 & 106.842 & 88.0996 & 4 & 3.814 & 4 & 78.302 & -3.67 & 4.69335 & 52 & 0 \\
\hline Apomorphine & 52.483 & 253.32 & 235.175 & 74.119 & 6 & 2.7217 & 0 & 173.019 & -3.04 & 2.29605 & 42 & 2 \\
\hline Arbekacin & 297.289 & 552.72 & 495.513 & 129.083 & 26 & -6.0054 & 10 & 405.668 & -1.13 & 11.7328 & 104.333 & 5 \\
\hline ascorbic acid & 176.14 & 108.667 & 67.2534 & 36.4585 & 10 & -2.4403 & 2 & 107.217 & 0.14 & 2.55413 & 43.6667 & 4 \\
\hline Astemizole & 32.325 & 458.63 & 459.619 & 133.862 & 4 & 5.1724 & 8 & 345.74 & -5.7 & 7.40812 & 70.5 & 0 \\
\hline Atenolol & 266.38 & 193.726 & 109.65 & 73.5041 & 7 & 0.560801 & 8 & 84.583 & -2.79 & 6.65708 & 47.1667 & 1 \\
\hline atorvastatin & 558.7 & 266.103 & 189.683 & 155.92 & 9 & 5.3024 & 12 & 111.787 & -5.95 & 10.0944 & 103.167 & 3 \\
\hline Atovaquone & 366.86 & 189.313 & 112.775 & 106.199 & 4 & 2.8196 & 2 & 54.37 & -5.14 & 4.51288 & 59.3111 & 1 \\
\hline atropine & 289.41 & 198.438 & 135.628 & 80.8156 & 5 & 1.712 & 5 & 49.771 & -2.06 & 4.3811 & 46.6667 & 1 \\
\hline Azathioprine & 277.29 & 150.59 & 86.4799 & 70.9782 & 7 & 0.4465 & 2 & 118.117 & -2.41 & 3.94833 & 49.4667 & 0 \\
\hline Azithromycin & 749.121 & 575.36 & 200.208 & 194.104 & 19 & 2.3016 & 7 & 180.091 & -3.16 & 15.721 & 122.917 & 5 \\
\hline
\end{tabular}


Table 1 - (continuation).

\begin{tabular}{|c|c|c|c|c|c|c|c|c|c|c|c|c|}
\hline Name of drug & $\begin{array}{c}\text { Molecular } \\
\text { mass }\end{array}$ & $\begin{array}{c}\text { Molecular } \\
\text { surface } \\
\text { area }\end{array}$ & $\begin{array}{c}\text { Molecular } \\
\text { volume }\end{array}$ & $\begin{array}{l}\text { Molecular } \\
\text { refractivity }\end{array}$ & $\begin{array}{c}\text { Total } \mathrm{H} \\
\text { bond } \\
\text { counting }\end{array}$ & $\log P$ & $\begin{array}{l}\text { Rotable } \\
\text { bonds }\end{array}$ & TPSA & $\log S$ & $\begin{array}{c}\text { Shape } \\
\text { flexibility } \\
\text { index }\end{array}$ & $\begin{array}{l}\text { Sum of } \\
\text { E-states } \\
\text { indices }\end{array}$ & $\begin{array}{l}\text { Hydroxyl } \\
\text { groups }\end{array}$ \\
\hline azosemide & 370.86 & 160.712 & 101.5 & 93.2608 & 8 & 1.2139 & 5 & 126.659 & -3.59 & 5.5104 & 55.7611 & 0 \\
\hline aztreonam & 435.47 & 191.872 & 127.033 & 108 & 13 & -4.461 & 7 & 203.165 & -4.06 & 6.81075 & 87.8667 & 1 \\
\hline Baclofen & 213.68 & 135.225 & 74.4967 & 54.829 & 5 & 1.5 & 4 & 63.322 & -2.48 & 4.07015 & 35.6444 & 1 \\
\hline Barnidipine & 113.699 & 477.56 & 428.691 & 131.543 & 8 & 2.3799 & 8 & 355.436 & -5.1 & 7.74161 & 87.5 & 0 \\
\hline benazepril & 424.54 & 219.229 & 144.424 & 115.227 & 8 & 3.2976 & 10 & 95.94 & -4.61 & 8.19548 & 76.6667 & 1 \\
\hline benzylpenicillin & 334.42 & 183.527 & 111.144 & 84.4332 & 6 & 0.912 & 4 & 86.706 & -3.07 & 4.13633 & 59.55 & 1 \\
\hline Betamethasone & 392.51 & 188.988 & 110.988 & 102.585 & 8 & 1.7117 & 2 & 94.826 & -3.89 & 4.15069 & 74.8333 & 3 \\
\hline Betaxolol & 307.48 & 215.583 & 145.313 & 88.6378 & 6 & 2.4993 & 11 & 50.723 & -4.01 & 7.32635 & 45.3333 & 1 \\
\hline Biperiden & 23.47 & 311.51 & 319.018 & 97.0148 & 3 & 3.47 & 5 & 260.3 & -4.86 & 4.29475 & 42.4167 & 1 \\
\hline bornaprine & 329.53 & 203.282 & 135.469 & 98.2855 & 3 & 4.0985 & 9 & 29.543 & -5.11 & 5.72484 & 47.25 & 0 \\
\hline Bretylium & 243.19 & 142.67 & 79.8429 & 59.77 & 0 & -0.903 & 3 & 0 & -6.26 & 3.50955 & 22.8958 & 0 \\
\hline Bromazepam & 316.17 & 144.167 & 87.9727 & 75.2043 & 4 & 2.1236 & 1 & 54.354 & -3.9 & 3.76437 & 42.0625 & 0 \\
\hline bromocriptine & 654.671 & 294.272 & 218.108 & 165.511 & 9 & 3.9423 & 5 & 118.207 & -3.88 & 7.03448 & 95.0625 & 1 \\
\hline Bucindolol & 81.07 & 363.5 & 361.705 & 106.912 & 7 & 3.1969 & 8 & 282.416 & -4.69 & 6.0447 & 60.4167 & 1 \\
\hline Budesonide & 93.07 & 430.59 & 379.029 & 116.202 & 8 & 2.4653 & 4 & 331.985 & -3.97 & 4.90939 & 72.25 & 2 \\
\hline Bumetanide & 364.45 & 189.136 & 117.647 & 93.5013 & 8 & 2.3317 & 8 & 118.725 & -4.15 & 6.40214 & 67.8167 & 1 \\
\hline bumetanide & 364.45 & 189.136 & 117.647 & 93.5013 & 8 & 2.3317 & 8 & 118.725 & -4.15 & 6.40214 & 67.8167 & 1 \\
\hline buprenorphine & 467.71 & 229.941 & 159.036 & 131.398 & 7 & 3.7834 & 5 & 62.162 & -4.44 & 4.22981 & 66.75 & 2 \\
\hline Bupropion & 239.77 & 142.152 & 89.5368 & 67.697 & 3 & 2.7496 & 4 & 29.098 & -3.54 & 4.11091 & 34.3944 & 0 \\
\hline Buspirone & 385.57 & 244.933 & 142.61 & 108.137 & 5 & 1.5978 & 6 & 69.64 & -2.82 & 6.75123 & 59.25 & 0 \\
\hline butorphanol & 327.51 & 181.682 & 123.075 & 95.6328 & 5 & 3.5487 & 2 & 43.694 & -3.31 & 3.39039 & 46.6667 & 2 \\
\hline Caffeine & 217.31 & 130.857 & 89.6923 & 54.7325 & 5 & 0.2974 & 3 & 57.608 & -1.68 & 4.09572 & 37.2222 & 1 \\
\hline Calcitirol & 416.71 & 257.327 & 146.587 & 126.531 & 6 & 4.5268 & 8 & 60.684 & -4.8 & 7.36659 & 62.1667 & 3 \\
\hline camazepam & 371.85 & 181.518 & 115.635 & 98.6319 & 4 & 3.3971 & 3 & 62.216 & -4.31 & 5.58356 & 60.8111 & 0 \\
\hline Candesartan & 440.5 & 239.744 & 139.441 & 125.437 & 9 & 4.9539 & 7 & 118.826 & -4.77 & 6.75684 & 76.6667 & 1 \\
\hline capreomycin la & 668.831 & 353.26 & 254.659 & 162.204 & 26 & -9.636 & 11 & 378.42 & -2.65 & 17.3594 & 136.5 & 1 \\
\hline captopril & 217.31 & 130.857 & 89.6923 & 54.7325 & 5 & 0.2974 & 3 & 57.608 & -1.68 & 4.09572 & 37.2222 & 1 \\
\hline Carbamazepine & 236.29 & 141.531 & 75.8141 & 71.888 & 2 & 2.7227 & 0 & 48.028 & -3.19 & 2.5525 & 41.3333 & 0 \\
\hline Carbimazole & 36.17 & 186.25 & 194.429 & 49.5677 & 3 & 1.6421 & 2 & 125.627 & -1.77 & 3.04622 & 28.6 & 0 \\
\hline carfecillin & 302.26 & 160.489 & 98.4169 & 77.282 & 12 & 3.1766 & 5 & 139.782 & -3.59 & 5.10618 & 69.3333 & 4 \\
\hline Carvedilol & 406.52 & 270.429 & 153.152 & 115.638 & 8 & 2.9421 & 10 & 75.748 & -4.96 & 6.86052 & 64.5 & 1 \\
\hline Cefacetrile & 136.8 & 339.35 & 306.125 & 77.9676 & 9 & -2.4602 & 6 & 235.52 & -2.15 & 5.40026 & 70.3 & 1 \\
\hline Cefadroxil & 363.42 & 193.566 & 114.318 & 90.6727 & 10 & -0.8287 & 4 & 132.957 & -2.96 & 4.86907 & 69.3 & 2 \\
\hline Cefazolin & 454.54 & 236.705 & 137.34 & 113.659 & 11 & -1.6174 & 7 & 156.1 & -2.97 & 7.58985 & 73.2333 & 1 \\
\hline cefetamet pivoxil(globocef) & 511.62 & 238.956 & 161.702 & 124.856 & 11 & 1.2192 & 11 & 162.53 & -4.05 & 9.02258 & 88.1833 & 0 \\
\hline Cefixime & 453.48 & 212.396 & 128.462 & 106.861 & 13 & -0.9987 & 9 & 184.518 & -3.64 & 7.53171 & 86.9333 & 2 \\
\hline Cefodizime & 197.41 & 584.7 & 495.311 & 141.32 & 14 & -0.1411 & 11 & 396.848 & -2.79 & 9.98652 & 96.7 & 2 \\
\hline Cefoxitin & 148.27 & 427.48 & 341.143 & 100.836 & 10 & -0.5798 & 8 & 300.491 & -3.32 & 6.31343 & 77.5167 & 1 \\
\hline ceftizoxime & 383.43 & 176.916 & 110.151 & 91.8494 & 10 & -0.8751 & 6 & 147.219 & -3.22 & 5.89243 & 68.1 & 1 \\
\hline cefuroxime & 424.42 & 206.164 & 128.6 & 97.1708 & 12 & -1.6699 & 9 & 173.772 & -3.17 & 6.92599 & 83.6333 & 1 \\
\hline cefuroximeaxetil & 510.52 & 239.099 & 159.045 & 117.035 & 13 & -1.5715 & 13 & 189.08 & -3.54 & 9.40496 & 98.6333 & 0 \\
\hline Cephalexin & 347.42 & 185.242 & 117.747 & 88.9786 & 8 & -0.5443 & 4 & 112.729 & -3.07 & 4.65839 & 63.6333 & 1 \\
\hline Cephalothin & 113.01 & 396.46 & 330.949 & 95.7433 & 8 & -1.3491 & 7 & 275.989 & -3.88 & 5.90142 & 70.1 & 1 \\
\hline Cephradine & 349.44 & 193.345 & 107.607 & 92.0006 & 8 & -1.0473 & 4 & 112.729 & -2.65 & 4.83975 & 62.6333 & 1 \\
\hline cetirizine & 388.93 & 214.25 & 145.458 & 106.867 & 6 & 3.477 & 8 & 53.009 & -3.77 & 7.37913 & 58.3111 & 1 \\
\hline
\end{tabular}


Table 1 - (continuation).

\begin{tabular}{|c|c|c|c|c|c|c|c|c|c|c|c|c|}
\hline Name of drug & $\begin{array}{c}\text { Molecular } \\
\text { mass }\end{array}$ & $\begin{array}{c}\text { Molecular } \\
\text { surface } \\
\text { area }\end{array}$ & $\begin{array}{c}\text { Molecular } \\
\text { volume }\end{array}$ & $\begin{array}{l}\text { Molecular } \\
\text { refractivity }\end{array}$ & $\begin{array}{c}\text { Total } \mathrm{H} \\
\text { bond } \\
\text { counting }\end{array}$ & $\log P$ & $\begin{array}{c}\text { Rotable } \\
\text { bonds }\end{array}$ & TPSA & $\log S$ & \begin{tabular}{|c|} 
Shape \\
flexibility \\
index
\end{tabular} & $\begin{array}{c}\text { Sum of } \\
\text { E-states } \\
\text { indices }\end{array}$ & $\begin{array}{c}\text { Hydroxyl } \\
\text { groups }\end{array}$ \\
\hline chlorambucil & 304.24 & 166.488 & 116.076 & 78.9619 & 3 & 3.6693 & 9 & 40.537 & -3.59 & 8.06516 & 41.1222 & 1 \\
\hline chloramphenicol & 323.15 & 157.676 & 109.428 & 73.2007 & 8 & 1.1709 & 5 & 115.378 & -2.85 & 6.4702 & 58.6222 & 2 \\
\hline Chlordiazepoxide & 299.78 & 184.553 & 105.434 & 80.762 & 3 & 0.731 & 2 & 53.144 & -4.18 & 4.10979 & 45.3111 & 0 \\
\hline chlorothiazide & 295.73 & 119.95 & 72.0424 & 60.8266 & 7 & -0.06 & 1 & 118.698 & -2.85 & 2.9919 & 52.1111 & 0 \\
\hline chlorpromazine & 318.89 & 175.926 & 115.606 & 93.8587 & 1 & 3.7952 & 4 & 8.172 & -4.88 & 4.55038 & 36.7778 & 0 \\
\hline chlorpropamide & 276.76 & 148.693 & 89.5262 & 65.4322 & 5 & 1.8688 & 4 & 75.267 & -3.25 & 5.07249 & 45.6278 & 0 \\
\hline chlorthalidone & 338.78 & 154.425 & 90.9938 & 80.8027 & 7 & 2.1436 & 2 & 109.491 & -3.81 & 3.35018 & 60.3778 & 1 \\
\hline cicaprost & 374.52 & 256.566 & 170.799 & 105.169 & 8 & 2.6665 & 13 & 86.989 & -4.23 & 8.53789 & 66.3333 & 3 \\
\hline cidofovir & 279.22 & 140.876 & 95.8058 & 59.8675 & 11 & -0.6864 & 6 & 147.909 & -1.37 & 5.67843 & 57.9722 & 3 \\
\hline cimetidine & 256.42 & 174.992 & 112.204 & 70.4443 & 8 & 0.585801 & 8 & 88.893 & -2.59 & 7.65293 & 36.4667 & 0 \\
\hline Cinacalcet & 12.03 & 357.45 & 335.378 & 100.116 & 2 & 6.2948 & 6 & 275.335 & -6.81 & 5.62113 & 65.9167 & 0 \\
\hline Ciprofloxacin & 331.38 & 176.144 & 125.779 & 89.8039 & 6 & -1.6602 & 3 & 74.569 & -1.95 & 3.80369 & 62.5 & 1 \\
\hline cisapride & 466 & 252.289 & 171.582 & 122.437 & 7 & 2.2463 & 9 & 86.061 & -4.59 & 9.12404 & 74.6444 & 0 \\
\hline citalopram & 324.43 & 177.294 & 120.095 & 94.2915 & 3 & 3.5895 & 5 & 36.264 & -4.74 & 4.6575 & 55.5833 & 0 \\
\hline Clavulanate & 199.18 & 171.481 & 97.1736 & 43.9836 & 7 & -1.6561 & 3 & 87.07 & 0.23 & 2.19789 & 44.1667 & 2 \\
\hline Clindamycin & 425.04 & 228.085 & 130.532 & 105.77 & 10 & 1.2095 & 7 & 102.254 & -2.14 & 8.82537 & 62.6111 & 3 \\
\hline Clodronate & 120.71 & 242.87 & 161.058 & 35.1764 & 8 & 0.721 & 2 & 136.379 & -1.16 & 2.94435 & 44.4833 & 2 \\
\hline clofibrate & 242.72 & 144.973 & 90.7803 & 62.1337 & 3 & 2.968 & 5 & 35.539 & -3.92 & 4.47237 & 37.0611 & 0 \\
\hline Clomethiazolel & 12.89 & 161.66 & 169.872 & 42.7201 & 1 & 1.3503 & 2 & 114.028 & -1.59 & 3.00329 & 15.2778 & 0 \\
\hline Clonazepam & 315.73 & 166.483 & 98.3592 & 82.2399 & 5 & 2.7162 & 1 & 87.286 & -4.47 & 4.06957 & 56.9778 & 0 \\
\hline Clonidine & 295.73 & 119.95 & 72.0424 & 60.8266 & 7 & -0.06 & 1 & 118.698 & -2.85 & 2.9919 & 52.1111 & 0 \\
\hline clorazepate & 314.74 & 159.685 & 95.2244 & 80.8953 & 6 & 2.7482 & 2 & 78.761 & -4.1 & 4.11694 & 55.8111 & 1 \\
\hline Clorothiazide & 256.42 & 174.992 & 112.204 & 70.4443 & 8 & 0.585801 & 8 & 88.893 & -2.59 & 7.65293 & 36.4667 & 0 \\
\hline Cloxacillin & 435.91 & 208.993 & 122.132 & 106.044 & 8 & 2.1003 & 4 & 112.738 & -3.91 & 5.2568 & 72.5278 & 1 \\
\hline clozapine & 326.86 & 196.818 & 122.111 & 94.5489 & 3 & 3.3061 & 1 & 35.159 & -3.25 & 4.16383 & 42.8111 & 0 \\
\hline cocaine & 303.39 & 158.322 & 100.863 & 80.6618 & 5 & 1.9253 & 5 & 55.848 & -1.78 & 4.37987 & 51.8333 & 0 \\
\hline Corticosterone & 346.51 & 167.149 & 119.238 & 96.0927 & 6 & 2.1476 & 2 & 74.598 & -3.88 & 4.0056 & 58.1667 & 2 \\
\hline Coumarin & 30.21 & 146.15 & 152.63 & 41.5486 & 2 & 1.8198 & 0 & 93.0943 & -2.16 & 1.33348 & 27.5 & 0 \\
\hline Cromog & 173.72 & 468.39 & 385.527 & 114.107 & 14 & -0.3921 & 8 & 321.633 & -4.12 & 6.67533 & 100.333 & 3 \\
\hline Cyclic-HPMPC & 116.69 & 261.2 & 229.004 & 56.3753 & 8 & -0.3894 & 2 & 172.916 & -1.2 & 3.82362 & 49.4722 & 1 \\
\hline Cyclophosphamide & 261.11 & 142.514 & 81.4258 & 58.4781 & 5 & 0.782501 & 5 & 41.57 & -1.24 & 5.75129 & 28.4278 & 0 \\
\hline cycloserine & 102.11 & 77.8568 & 43.7337 & 21.8488 & 5 & -1.4009 & 0 & 64.335 & 0.93 & 1.05592 & 21.5 & 0 \\
\hline cyproterone acetate & 416.98 & 195.653 & 134.541 & 111.902 & 4 & 2.6109 & 3 & 60.447 & -5.44 & 4.01408 & 64.0611 & 0 \\
\hline Cytarabine & 243.25 & 168.079 & 94.7432 & 54.7448 & 10 & -1.374 & 2 & 130.838 & -0.12 & 3.62125 & 51.6667 & 3 \\
\hline dalfopristin & 690.941 & 282.837 & 214.175 & 182.712 & 13 & 2.3106 & 7 & 176.423 & -3.98 & 15.2975 & 121.983 & 1 \\
\hline Dapsone & 24 & 170.017 & 92.4548 & & 4 & 1.3069 & 2 & 86.188 & -2.94 & 2.97388 & 44.9833 & 0 \\
\hline delavirdine & 470.76 & 251.059 & 183.79 & 125.248 & 12 & -0.4726 & 6 & 110.426 & -3.73 & 7.91119 & 68.8167 & 0 \\
\hline Desipramine & 266.42 & 171.417 & 107.483 & 85.4133 & 2 & 3.6205 & 4 & 15.265 & -3.83 & 4.14258 & 36.6667 & 0 \\
\hline Desmethyldiazepam(nordazepam & 270.73 & 157.388 & 94.842 & 74.9152 & 3 & 2.7626 & 1 & 41.462 & -4.09 & 3.41029 & 41.3111 & 0 \\
\hline Desogestrel & 20.23 & 310.52 & 313.124 & 95.7331 & 2 & 4.2569 & 2 & 257.85 & -5.01 & 3.54334 & 44.1667 & 1 \\
\hline Dexamethasone & 392.51 & 178.733 & 126.416 & 102.585 & 8 & 1.7117 & 2 & 94.826 & -3.89 & 4.15069 & 74.8333 & 3 \\
\hline Diacetylmorphine & 65.08 & 383.48 & 341.999 & 102.95 & 6 & 1.6676 & 4 & 284.271 & -3.53 & 4.106 & 63.0833 & 0 \\
\hline Diazepam & 284.76 & 158.56 & 94.8058 & 79.8119 & 2 & 3.0091 & 1 & 32.673 & -4.37 & 3.64372 & 42.8111 & 0 \\
\hline Diazoxide & 230.68 & 127.223 & 72.3984 & & 4 & 1.1355 & 0 & 58.533 & -2.68 & 2.33397 & 35.7944 & 0 \\
\hline Diclofenac & 296.16 & 165.705 & 96.165 & 75.1658 & 4 & 3.8258 & 4 & 49.326 & -4.82 & 4.55626 & 43.6222 & 1 \\
\hline
\end{tabular}


Table 1 - (continuation).

\begin{tabular}{|c|c|c|c|c|c|c|c|c|c|c|c|c|}
\hline Name of drug & $\begin{array}{c}\text { Molecular } \\
\text { mass }\end{array}$ & $\begin{array}{c}\text { Molecular } \\
\text { surface } \\
\text { area }\end{array}$ & $\begin{array}{c}\text { Molecular } \\
\text { volume }\end{array}$ & $\begin{array}{l}\text { Molecular } \\
\text { refractivity }\end{array}$ & $\begin{array}{c}\text { Total } \mathrm{H} \\
\text { bond } \\
\text { counting }\end{array}$ & $\log P$ & $\begin{array}{l}\text { Rotable } \\
\text { bonds }\end{array}$ & TPSA & $\log S$ & $\begin{array}{c}\text { Shape } \\
\text { flexibility } \\
\text { index }\end{array}$ & $\begin{array}{l}\text { Sum of } \\
\text { E-states } \\
\text { indices }\end{array}$ & $\begin{array}{c}\text { Hydroxyl } \\
\text { groups }\end{array}$ \\
\hline dicloxacillin & 470.35 & 198.158 & 125.083 & 110.849 & 8 & 2.6183 & 4 & 112.738 & -4.2 & 5.6832 & 73.5056 & 1 \\
\hline didanosine & 235.25 & 137.149 & 86.5179 & 55.814 & 7 & -0.0384 & 2 & 93.042 & -1.53 & 3.24819 & 43.6667 & 1 \\
\hline Diflunisal & 250.21 & 151.631 & 81.4506 & 60.0795 & 5 & 3.424 & 2 & 57.527 & -3.55 & 3.18149 & 58.6667 & 2 \\
\hline Digitoxin & 765.051 & 386.341 & 235.484 & 191.714 & 18 & 3.5857 & 7 & 182.849 & -4.42 & 11.0006 & 122.75 & 5 \\
\hline digoxin & 781.051 & 374.8 & 280.137 & 193.229 & 20 & 2.6723 & 7 & 203.077 & -3.79 & 11.2146 & 128.583 & 6 \\
\hline dihydrocodeine & 301.42 & 166.327 & 112.619 & 83.5656 & 5 & 1.5062 & 1 & 41.134 & -2.1 & 2.65139 & 43.75 & 1 \\
\hline Dihydroergosine & 118.21 & 549.74 & 506.427 & 148.448 & 9 & 2.8029 & 4 & 413.303 & -3.17 & 6.04256 & 90.1667 & 1 \\
\hline Dihydroergotamine & 118.21 & 583.75 & 517.093 & 159.393 & 9 & 3.2933 & 4 & 442.516 & -3.41 & 6.19692 & 96.5 & 1 \\
\hline Dilevalol & 69.55 & 327.46 & 347.497 & 95.7518 & 7 & 3.0614 & 8 & 255.434 & -4.52 & 6.67046 & 57 & 2 \\
\hline Diltiazem & 414.56 & 241.128 & 137.629 & 114.339 & 5 & 2.3969 & 7 & 59.086 & -4.39 & 7.20953 & 65.3 & 0 \\
\hline Dimercaprol & 20.23 & 124.23 & 129.549 & 32.7701 & 4 & 0.0923 & 2 & 89.5857 & -1.65 & 4.25871 & 12.7778 & 1 \\
\hline diphenhydramin & 255.39 & 169.041 & 104.317 & 79.927 & 2 & 3.6159 & 6 & 12.472 & -3.53 & 5.13142 & 37.1667 & 0 \\
\hline Dirithromycin & 196.35 & 835.221 & 711.467 & 212.949 & 21 & 2.2609 & 12 & 659.265 & -3.56 & 17.5766 & 133.583 & 4 \\
\hline disopyramide & 339.53 & 178.947 & 119.742 & 102.418 & 4 & 3.5272 & 8 & 59.224 & -3.84 & 6.80283 & 53.9167 & 0 \\
\hline Distigmine & 66.86 & 416.58 & 451.125 & 115.08 & 4 & 2.7998 & 11 & 317.181 & -6.02 & 10.0184 & 68.6667 & 0 \\
\hline disulfiram & 296.56 & 157.899 & 113.369 & 89.0288 & 2 & 4.7516 & 9 & 6.476 & -4.37 & 10.2814 & 25.1333 & 0 \\
\hline Dixyrazine & 40.87 & 427.66 & 412.296 & 126.856 & 5 & 2.9732 & 9 & 323.134 & -4.12 & 7.81754 & 57.1333 & 1 \\
\hline Dofetilide & 441.61 & 263.236 & 146.878 & 114.017 & 8 & 1.3337 & 11 & 104.81 & -4.35 & 8.31706 & 73.8 & 0 \\
\hline dolasetron & 332.49 & 204.078 & 141.688 & 89.4666 & 6 & 1.2299 & 3 & 62.405 & -3.09 & 3.79648 & 49.6667 & 0 \\
\hline Doxepin & 279.41 & 192.72 & 104.137 & 89.0687 & 2 & 3.5655 & 4 & 12.472 & -3.94 & 4.18303 & 40.3333 & 0 \\
\hline doxorubicin & 543.57 & 249.729 & 176.705 & 134.019 & 18 & 0.171802 & 5 & 206.074 & -2.67 & 7.0384 & 109.417 & 5 \\
\hline doxycycline & 444.48 & 210.382 & 141.815 & 113.125 & 15 & -2.8004 & 2 & 181.614 & -2.85 & 5.10331 & 93.5833 & 5 \\
\hline eflornithine & 182.2 & 111.161 & 71.7719 & 37.7328 & 7 & -1.0467 & 5 & 89.345 & -0.56 & 4.22091 & 45.75 & 1 \\
\hline enalapril & 376.5 & 217.1 & 145.306 & 99.5722 & 8 & 2.101 & 10 & 95.94 & -3.25 & 8.24443 & 68.6667 & 1 \\
\hline enalaprilat & 348.44 & 195.618 & 128.714 & 90.0551 & 9 & 1.7268 & 8 & 106.934 & -2.6 & 6.95061 & 67.6667 & 2 \\
\hline Encainide & 352.52 & 228.206 & 129.763 & 105.486 & 4 & 3.8831 & 6 & 41.57 & -4.94 & 6.55577 & 53.6667 & 0 \\
\hline entacapone & 305.32 & 161.047 & 104.954 & 80.395 & 8 & 1.5266 & 5 & 130.381 & -3.58 & 6.03161 & 68.5 & 2 \\
\hline Epanolol & 114.61 & 369.46 & 375.117 & 101.212 & 10 & 1.5011 & 10 & 273.721 & -3.78 & 8.57852 & 69.1667 & 2 \\
\hline Epirubicin & 206.08 & 557.6 & 475.978 & 138.568 & 18 & 0.104301 & 6 & 393.173 & -2.81 & 7.5028 & 110.917 & 5 \\
\hline Eprosartan & 92.42 & 424.55 & 352.539 & 118.755 & 7 & 4.6711 & 11 & 324.662 & -5.89 & 8.0494 & 72.4667 & 2 \\
\hline Estradiol & 40.46 & 272.42 & 270.852 & 79.6175 & 4 & 4.0079 & 0 & 208.477 & -4.11 & 2.7726 & 40.5833 & 2 \\
\hline Estradiol_17-valerate & 46.53 & 356.55 & 359.872 & 102.598 & 4 & 5.5578 & 5 & 286.252 & -5.76 & 4.95701 & 53.25 & 1 \\
\hline ethambutol & 204.36 & 156.144 & 104.244 & 57.8872 & 8 & 0.290401 & 9 & 64.51 & -1.43 & 9.28976 & 32.6667 & 2 \\
\hline ethanol & 46.08 & 63.2038 & 32.2323 & 13.0093 & 2 & 0.0772 & 0 & 20.228 & 1.1 & 1.93387 & 9.5 & 1 \\
\hline ethinylestradiol & 296.44 & 170.001 & 111.419 & 87.0873 & 4 & 4.0174 & 1 & 40.456 & -4.64 & 2.99027 & 47 & 2 \\
\hline Etidronate & 135.28 & 206.04 & 158.803 & 33.3638 & 12 & 0.4419 & 2 & 124.507 & -1.25 & 2.70093 & 47.8611 & 5 \\
\hline etoposide & 588.61 & 280.328 & 195.188 & 138.584 & 16 & 0.918701 & 5 & 160.861 & -2.78 & 7.41546 & 103.333 & 3 \\
\hline famciclovir & 321.38 & 188.357 & 127.443 & 81.5349 & 8 & -0.6945 & 9 & 122.242 & -2.39 & 7.09464 & 59.6667 & 0 \\
\hline Famotidine & 337.48 & 203.802 & 121.412 & 80.8407 & 9 & -0.6491 & 9 & 175.854 & -3.1 & 8.1158 & 53.75 & 0 \\
\hline Felbamate & 238.27 & 169.214 & 90.6974 & 59.5868 & 6 & 0.7793 & 7 & 104.656 & -2.51 & 5.44157 & 48.3333 & 0 \\
\hline felodipine & 384.28 & 187.036 & 123.348 & 99.1964 & 5 & 1.6672 & 6 & 64.637 & -4.73 & 6.5428 & 57.9555 & 0 \\
\hline Femoxetine & 21.71 & 311.46 & 312.271 & 93.7979 & 3 & 3.5335 & 5 & 248.059 & -4.41 & 5.44182 & 44.6667 & 0 \\
\hline fenclofenac & 297.14 & 151.737 & 90.4496 & 73.216 & 4 & 4.1425 & 4 & 46.532 & -4.68 & 4.55626 & 44.6222 & 1 \\
\hline fenoterol & 303.39 & 175.572 & 117.268 & 84.1362 & 10 & 2.7878 & 6 & 92.99 & -3.27 & 5.65183 & 56.5 & 4 \\
\hline fentanyl & 336.52 & 206.673 & 134.176 & 103.482 & 2 & 3.7746 & 6 & 23.547 & -4.15 & 6.3729 & 49.8333 & 0 \\
\hline
\end{tabular}


Table 1 - (continuation).

\begin{tabular}{|c|c|c|c|c|c|c|c|c|c|c|c|c|}
\hline Name of drug & $\begin{array}{c}\text { Molecular } \\
\text { mass }\end{array}$ & $\begin{array}{c}\text { Molecular } \\
\text { surface } \\
\text { area }\end{array}$ & $\begin{array}{c}\text { Molecular } \\
\text { volume }\end{array}$ & $\begin{array}{l}\text { Molecular } \\
\text { refractivity }\end{array}$ & $\begin{array}{c}\text { Total } \mathrm{H} \\
\text { bond } \\
\text { counting }\end{array}$ & $\log P$ & $\begin{array}{c}\text { Rotable } \\
\text { bonds }\end{array}$ & TPSA & $\log S$ & $\begin{array}{c}\text { Shape } \\
\text { flexibility } \\
\text { index }\end{array}$ & $\begin{array}{l}\text { Sum of } \\
\text { E-states } \\
\text { indices }\end{array}$ & $\begin{array}{c}\text { Hydroxyl } \\
\text { groups }\end{array}$ \\
\hline Fexofenadine & 80.99 & 501.72 & 515.169 & 147.782 & 8 & 6.2599 & 10 & 399.004 & -5.28 & 8.60357 & 83 & 3 \\
\hline finasteride & 372.61 & 201.232 & 137.426 & 108.194 & 4 & 2.9957 & 2 & 58.196 & -5.27 & 4.47274 & 55.75 & 0 \\
\hline Flecainide & 414.39 & 202.762 & 124.572 & 87.8977 & 6 & 2.9815 & 7 & 59.593 & -4.11 & 7.81195 & 94 & 0 \\
\hline Fluconazole & 306.31 & 162.636 & 94.8887 & 76.6249 & 6 & 1.2158 & 5 & 81.664 & -2.34 & 4.96568 & 61.25 & 1 \\
\hline Flucytosine & 129.11 & 95.6792 & 50.4012 & 28.3514 & 4 & -0.9747 & 0 & 71.777 & -0.09 & 1.63973 & 31.5 & 0 \\
\hline flumazenil & 303.32 & 164.789 & 106.688 & 75.3538 & 4 & 1.345 & 3 & 64.44 & -2.46 & 3.94963 & 59.1667 & 0 \\
\hline Flunisolide & 93.07 & 434.55 & 349.895 & 111.977 & 8 & 1.8116 & 2 & 325.551 & -4.07 & 4.38391 & 79 & 2 \\
\hline Flunitrazepam & 313.31 & 166.912 & 101.855 & 82.5482 & 4 & 2.5842 & 1 & 78.497 & -4.56 & 4.0657 & 65.1667 & 0 \\
\hline Fluorouracil & 130.09 & 94.7107 & 50.8243 & 27.3802 & 4 & -1.0593 & 0 & 65.724 & -0.43 & 1.37263 & 34 & 0 \\
\hline Fluoxetine & 309.36 & 197.862 & 104.255 & 80.3675 & 3 & 4.1937 & 6 & 21.261 & -5.26 & 5.5746 & 60.5833 & 0 \\
\hline Fluphenazine & 437.57 & 235.138 & 141.516 & 117.37 & 4 & 3.5182 & 6 & 31.638 & -4.36 & 6.69367 & 73.7167 & 1 \\
\hline Flurbiprofen & 244.28 & 165.219 & 91.9894 & 67.2927 & 3 & 4.0638 & 3 & 37.299 & -3.99 & 3.53229 & 48.6667 & 1 \\
\hline Fluticasone_propionate & 68 & 500.62 & 398.985 & 121.867 & 6 & 2.8691 & 6 & 367.562 & -4.64 & 6.26109 & 94.9667 & 1 \\
\hline Fluvastatin & 411.51 & 200.683 & 146.599 & 114.854 & 7 & 3.8804 & 9 & 82.689 & -4.97 & 6.94553 & 77.6667 & 3 \\
\hline Fluvoxamine & 318.38 & 179.142 & 4.983 & & 5 & 2.6885 & 10 & 56.855 & -4.64 & 8.52913 & 63.25 & 0 \\
\hline Foscarnet & 126.01 & 72.4961 & 38.4522 & 18.4995 & 8 & 0.2579 & 1 & 94.826 & -0.88 & 1.65931 & 33.9722 & 3 \\
\hline fosfomycin & 138.07 & 87.4929 & 54.3809 & 25.2061 & 6 & 0.3208 & 1 & 70.055 & -0.47 & 1.2033 & 27.4722 & 2 \\
\hline fosmidomycin & 183.12 & 109.876 & 73.4989 & 36.1213 & 8 & -0.2284 & 4 & 98.064 & -0.92 & 4.47563 & 40.8056 & 3 \\
\hline Frovatriptan & 70.91 & 243.34 & 245.072 & 71.3392 & 5 & 0.4988 & 2 & 169.781 & -3.3 & 2.77876 & 39.8333 & 0 \\
\hline Furosemide & 330.76 & 179.149 & 100.294 & 75.1956 & 8 & 0.9383 & 5 & 122.631 & -3.45 & 4.56793 & 60.1278 & 1 \\
\hline Gabapentin & 171.27 & 110.912 & 73.3154 & 46.3283 & 5 & 0.9552 & 3 & 63.322 & -1.6 & 3.23123 & 30.4167 & 1 \\
\hline Gallopamil & 73.2 & 484.7 & 503.534 & 139.567 & 7 & 4.6309 & 14 & 388.876 & -4.95 & 11.3151 & 75.75 & 0 \\
\hline gallopamil & 484.701 & 274.59 & 194.004 & 139.567 & 7 & 4.6309 & 14 & 73.2 & -4.95 & 11.3151 & 75.75 & 0 \\
\hline Ganciclovir & 254.26 & 141.169 & 90.0066 & 57.9173 & 10 & -0.9621 & 5 & 139.293 & -1.42 & 4.75762 & 52 & 2 \\
\hline gatifloxacin & 375.44 & 182.627 & 129.148 & & 7 & 1.0608 & 4 & 83.803 & -2.37 & 4.68983 & 69.5 & 1 \\
\hline Gemfibrozil & 250.37 & 177.309 & 94.8295 & 71.8187 & 4 & 4.3269 & 6 & 46.533 & -3.95 & 5.05297 & 42.9167 & 1 \\
\hline Gentamicin_C1 & 199.74 & 477.69 & 435.567 & 118.019 & 20 & -3.0525 & 7 & 362.763 & -1.58 & 9.08806 & 80.25 & 3 \\
\hline Gentamicin_C1a & 187.71 & 448.64 & 396.991 & 110.148 & 18 & -2.2722 & 6 & 339.279 & -1.53 & 8.30797 & 75.9167 & 3 \\
\hline Gl-147211 & 86.14 & 502.62 & 433.72 & 138.643 & 8 & 0.2797 & 3 & 351.036 & -3.02 & 5.84189 & 79.8333 & 0 \\
\hline glimepiride & 490.68 & 271.318 & 181.213 & 129.803 & 8 & 2.8182 & 7 & 124.674 & -4.11 & 8.88575 & 85.15 & 0 \\
\hline glipizide & 445.59 & 250.333 & 159.41 & 115.395 & 9 & 2.1228 & 7 & 130.149 & -4.43 & 8.66521 & 77.65 & 0 \\
\hline Glucosamine & 116.16 & 179.2 & 164.804 & 37.5809 & 11 & -2.1078 & 1 & 126.7 & 0.49 & 2.99364 & 39.6667 & 4 \\
\hline glyburide & 494.05 & 255.734 & 170.988 & 126.481 & 8 & 3.4985 & 8 & 113.599 & -5.38 & 9.13147 & 80.1278 & 0 \\
\hline Granisetron & 2.46 & 220.731 & 124.887 & & 4 & 1.7053 & 2 & 50.162 & -2.86 & 4.03979 & 46.6667 & 0 \\
\hline Guanethidine & & 198.36 & 227.704 & 59.5808 & 4 & 1.1125 & 4 & 168.976 & -2.29 & 6.24197 & 28.6667 & 0 \\
\hline guanoxan & 207.26 & 134.803 & 80.9848 & 54.9351 & 5 & 0.8751 & 3 & 80.37 & -2.19 & 3.38222 & 35.8333 & 0 \\
\hline Haloperidol & 375.9 & 212.425 & 126.051 & 102.591 & 4 & 3.3784 & 6 & 40.537 & -4.93 & 6.06954 & 60.3944 & 1 \\
\hline Hesperidin & 234.299 & 610.62 & 502.428 & 140.193 & 23 & -0.1597 & 7 & 427.654 & -2.36 & 9.57336 & 121 & 8 \\
\hline Hexobarbital & 236.3 & 153.405 & 80.8345 & 62.1823 & 5 & 2.2043 & 1 & 69.972 & -2.39 & 3.23404 & 44.9167 & 1 \\
\hline Hydralazine & 63.84 & 160.2 & 169.122 & 50.2778 & 5 & 1.7633 & 1 & 101.725 & -1.79 & 2.20038 & 27.5 & 0 \\
\hline Hydrochlorothiazide & 297.75 & 124.189 & 76.9855 & 61.3288 & 7 & -0.5292 & 1 & 118.361 & -2.12 & 2.9235 & 51.1111 & 0 \\
\hline hydrocortisone & 362.51 & 174.574 & 121.066 & 97.4 & 8 & 1.4307 & 2 & 94.826 & -3.26 & 4.0378 & 64.0833 & 3 \\
\hline Hydromorphone & 285.37 & 172.695 & 90.4839 & 77.8995 & 5 & 1.8775 & 0 & 49.771 & -1.81 & 2.19981 & 45.5833 & 1 \\
\hline hydroxyurea & 76.07 & 69.7511 & 34.2725 & 14.6204 & 5 & -0.8179 & 0 & 75.349 & 0.55 & 1.66684 & 21.1667 & 1 \\
\hline Hypericin & 155.51 & 504.46 & 394.345 & 137.591 & 14 & 4.6352 & 0 & 315.974 & -4.84 & 3.66703 & 102 & 6 \\
\hline
\end{tabular}


CLASSIFICATION OF ORAL BIOAVAILABILITY OF DRUGS BY MACHINE LEARNING APPROACHES

Table 1 - (continuation)

\begin{tabular}{|c|c|c|c|c|c|c|c|c|c|c|c|c|}
\hline Name of drug & $\begin{array}{c}\text { Molecular } \\
\text { mass }\end{array}$ & $\begin{array}{c}\text { Molecular } \\
\text { surface } \\
\text { area }\end{array}$ & $\begin{array}{c}\text { Molecular } \\
\text { volume }\end{array}$ & $\begin{array}{l}\text { Molecular } \\
\text { refractivity }\end{array}$ & $\begin{array}{c}\text { Total } \mathrm{H} \\
\text { bond } \\
\text { counting }\end{array}$ & $\log P$ & $\begin{array}{l}\text { Rotable } \\
\text { bonds }\end{array}$ & TPSA & $\log S$ & $\begin{array}{c}\text { Shape } \\
\text { flexibility } \\
\text { index }\end{array}$ & $\begin{array}{l}\text { Sum of } \\
\text { E-states } \\
\text { indices }\end{array}$ & $\begin{array}{c}\text { Hydroxyl } \\
\text { groups }\end{array}$ \\
\hline Ibandronate & 138.52 & 319.27 & 297.884 & 68.4749 & 13 & 1.2093 & 9 & 226.611 & -1.38 & 7.37629 & 60.8611 & 5 \\
\hline Ibuprofen & 206.31 & 153.199 & 77.7689 & 60.7319 & 3 & 3.8302 & 4 & 37.299 & -3.48 & 4.03629 & 36.1667 & 1 \\
\hline Idarubicin & 497.54 & 248.469 & 152.775 & 125.854 & 15 & 0.9801 & 3 & 176.616 & -2.81 & 5.95833 & 98.75 & 4 \\
\hline ifosfamide & 261.11 & 128.419 & 88.0822 & 58.4781 & 5 & 0.782501 & 5 & 41.57 & -1.24 & 5.75129 & 28.4278 & 0 \\
\hline Iloprost & 77.76 & 360.54 & 387.692 & 105.181 & 7 & 3.6387 & 12 & 290.194 & -4.62 & 8.08649 & 61.8333 & 3 \\
\hline Imipenem & 116.223 & 299.38 & 284.294 & 75.6864 & 8 & -2.3217 & 7 & 215.536 & -2.59 & 5.03617 & 54.8 & 2 \\
\hline imipramine & 280.45 & 179.112 & 114.95 & 90.708 & 1 & 3.9819 & 4 & 6.476 & -3.63 & 4.37209 & 38.1667 & 0 \\
\hline Indinavir & 613.881 & 293.576 & 175.142 & 175.86 & 11 & 3.4564 & 12 & 118.02 & -4.11 & 11.2399 & 99.9167 & 2 \\
\hline indomethacine & 357.81 & 186.946 & 117.183 & 94.6078 & 5 & 3.6415 & 4 & 68.538 & -5.17 & 4.83122 & 61.3111 & 1 \\
\hline Indoramin & 48.13 & 347.5 & 347.506 & 105.44 & 4 & 2.9043 & 5 & 255.231 & -4.6 & 5.25052 & 52.6667 & 0 \\
\hline Irabesartan & 428.59 & 232.354 & 140.078 & 127.733 & 6 & 5.0254 & 7 & 87.14 & -4.7 & 6.86568 & 66.4167 & 0 \\
\hline Irinotecan & 113.68 & 588.77 & 545.474 & 165.35 & 9 & -0.431 & 5 & 437.066 & -3.86 & 7.80905 & 96.4167 & 1 \\
\hline isoniazid & 137.16 & 100.952 & 55.4772 & 36.9343 & 5 & 0.0204 & 1 & 68.013 & -0.59 & 2.42411 & 27.8333 & 0 \\
\hline |soproterenol & 72.11 & 197.26 & 207.255 & 52.8903 & 8 & 1.6534 & 3 & 154.3 & -1.18 & 3.59787 & 38.1667 & 3 \\
\hline isoxicam & 335.36 & 163.303 & 100.08 & 83.7239 & 8 & -0.5591 & 2 & 112.74 & -3.2 & 3.98964 & 63.9833 & 1 \\
\hline isradipine & 371.43 & 173.237 & 116.502 & 100.082 & 8 & 0.6701 & 6 & 103.561 & -3.21 & 6.01531 & 66.6667 & 0 \\
\hline kanamycin & 254.3 & 149.652 & 90.8072 & 72.5156 & 4 & 3.4613 & 4 & 54.37 & -4.08 & 3.87529 & 49.6667 & 1 \\
\hline Ketamine & 237.75 & 149.284 & 79.9286 & 65.5534 & 3 & 3.1695 & 2 & 29.098 & -3.71 & 3.3114 & 33.0611 & 0 \\
\hline Ketoprofen & 254.3 & 149.652 & 90.8072 & 72.5156 & 4 & 3.4613 & 4 & 54.37 & -4.08 & 3.87529 & 49.6667 & 1 \\
\hline ketorolac & 255.29 & 152.027 & 92.5972 & 70.1941 & 4 & 1.7575 & 3 & 59.304 & -2.7 & 2.8899 & 48.6667 & 1 \\
\hline k-strophanthoside & 484.58 & 210.993 & 165.783 & 106.134 & 26 & -5.6338 & 6 & 282.624 & -0.72 & 8.98513 & 96.5 & 7 \\
\hline Labetalol & 328.45 & 212.64 & 126.241 & 93.9296 & 8 & 2.5865 & 8 & 95.577 & -4.75 & 6.63399 & 59 & 2 \\
\hline Lacidipine & 90.94 & 455.6 & 418.813 & 129.91 & 7 & 1.9456 & 12 & 356.306 & -5.31 & 9.19165 & 80.5833 & 0 \\
\hline lactulose & 342.34 & 172.281 & 126.059 & 68.7741 & 19 & -2.4695 & 5 & 189.526 & 0.36 & 5.92011 & 74.9167 & 8 \\
\hline Lamivudine & 229.28 & 134.793 & 70.7576 & 55.1051 & 6 & -0.0843 & 2 & 90.382 & -0.7 & 3.4781 & 39.1333 & 1 \\
\hline Lamotrigine & 256.11 & 131.017 & 75.79 & 66.6197 & 5 & 2.3937 & 1 & 90.722 & -2.72 & 3.91722 & 35.6222 & 0 \\
\hline Lansoprazole & 369.39 & 209.736 & 120.065 & 87.7582 & 5 & 2.8347 & 5 & 67.88 & -3.17 & 5.56233 & 71.6722 & 0 \\
\hline letrozole & 285.33 & 159.512 & 96.6924 & 85.133 & 4 & 3.3412 & 3 & 78.302 & -3.55 & 4.28678 & 53 & 0 \\
\hline Leucovorin & 473.5 & 269.587 & 150.618 & 114.367 & 15 & -1.4996 & 9 & 219.836 & -3.07 & 8.65707 & 99.6667 & 2 \\
\hline Levetiracetam & 170.24 & 128.821 & 66.6904 & 44.0785 & 3 & -0.7139 & 3 & 63.403 & 0.24 & 2.94331 & 32.6667 & 0 \\
\hline levodopa & 197.21 & 119.552 & 73.4903 & 48.5041 & 9 & 0.5835 & 3 & 103.778 & -1.78 & 3.3168 & 44.5 & 3 \\
\hline Levodopa & 197.21 & 124.493 & 72.9467 & 48.5041 & 9 & 0.5835 & 3 & 103.778 & -1.78 & 3.3168 & 44.5 & 3 \\
\hline levofloxacin & 361.41 & 181.481 & 121.275 & 94.7015 & 6 & 0.848201 & 2 & 75.014 & -1.87 & 4.24503 & 67.6667 & 1 \\
\hline levonorgestrel & 312.49 & 186.716 & 124.64 & 92.1184 & 3 & 2.8711 & 2 & 37.299 & -4.73 & 3.50421 & 48.1667 & 1 \\
\hline Lidocaine & 234.38 & 172.96 & 94.2785 & 72.1476 & 3 & 2.3815 & 5 & 32.336 & -2.6 & 5.32265 & 36.6667 & 0 \\
\hline lincomycin & 406.6 & 214.237 & 154.169 & 102.719 & 12 & 0.062801 & 7 & 122.148 & -1.14 & 8.50656 & 67.3 & 4 \\
\hline lisinopril & 405.55 & 216.988 & 149.153 & 107.367 & 11 & 1.4934 & 12 & 132.957 & -3.27 & 9.55958 & 75.6667 & 2 \\
\hline Lisuride & 51.365 & 338.5 & 333.846 & 101.811 & 4 & 1.721 & 3 & 246.922 & -3.38 & 4.33246 & 50.6667 & 0 \\
\hline Loracarbef & 349.8 & 184.423 & 113.249 & 86.6367 & 8 & -0.9616 & 4 & 112.729 & -3.03 & 4.61713 & 63.8111 & 1 \\
\hline Iorazepam & 321.17 & 152.688 & 92.4157 & 80.9184 & 5 & 3.3177 & 1 & 61.69 & -4.26 & 4.04774 & 48.1222 & 1 \\
\hline Lorcainide & 23.547 & 370.96 & 369.355 & 108.181 & 2 & 4.2371 & 5 & 294.924 & -4.69 & 6.46697 & 51.1444 & 0 \\
\hline Lormetazepam & 335.2 & 160.688 & 100.999 & 85.8151 & 4 & 3.5642 & 1 & 52.901 & -4.31 & 4.28476 & 49.6222 & 1 \\
\hline Iornoxicam & 371.83 & 169.005 & 103.373 & 88.5845 & 7 & -0.5009 & 2 & 99.598 & -3.93 & 4.43274 & 60.4278 & 1 \\
\hline Losartan & 422.96 & 209.252 & 131.327 & 123.184 & 7 & 5.0315 & 8 & 92.521 & -4.97 & 7.71104 & 62.6444 & 1 \\
\hline lovastatin & 404.6 & 238.19 & 166.929 & 113.181 & 6 & 3.7675 & 7 & 72.838 & -4.22 & 7.46643 & 65.6667 & 1 \\
\hline
\end{tabular}


Table 1 - (continuation)

\begin{tabular}{|c|c|c|c|c|c|c|c|c|c|c|c|c|}
\hline Name of drug & $\begin{array}{c}\text { Molecular } \\
\text { mass }\end{array}$ & $\begin{array}{c}\text { Molecular } \\
\text { surface } \\
\text { area }\end{array}$ & $\begin{array}{c}\text { Molecular } \\
\text { volume }\end{array}$ & $\begin{array}{l}\text { Molecular } \\
\text { refractivity }\end{array}$ & \begin{tabular}{|c} 
Total $\mathrm{H}$ \\
bond \\
counting
\end{tabular} & $\log P$ & $\begin{array}{l}\text { Rotable } \\
\text { bonds }\end{array}$ & TPSA & $\log S$ & $\begin{array}{c}\text { Shape } \\
\text { flexibility } \\
\text { index }\end{array}$ & $\begin{array}{c}\text { Sum of } \\
\text { E-states } \\
\text { indices }\end{array}$ & $\begin{array}{c}\text { Hydroxyl } \\
\text { groups }\end{array}$ \\
\hline Lynestrenol & 20.228 & 284.48 & 289.583 & 86.806 & 2 & 3.9223 & 1 & 235.388 & -4.89 & 3.05975 & 39.5 & 1 \\
\hline Mannitol & 121.368 & 182.2 & 170.334 & 38.4036 & 12 & -2.1984 & 5 & 132.652 & 0.1 & 5.30519 & 44.3333 & 6 \\
\hline Mebendazole & 295.32 & 192.453 & 109.433 & 79.7231 & 6 & 2.5231 & 4 & 84.086 & -3.87 & 4.15423 & 55.1667 & 0 \\
\hline Medifoxamine & 21.706 & 257.36 & 271.852 & 75.8301 & 3 & 3.7267 & 6 & 210.396 & -2.9 & 5.09523 & 39.1667 & 0 \\
\hline Medroxyprogesterone & 344.54 & 182.966 & 105.655 & 98.7468 & 4 & 3.3025 & 1 & 54.37 & -4.35 & 3.68622 & 54.5833 & 1 \\
\hline Melagatran & 148.608 & 429.58 & 416.119 & 114.786 & 10 & 0.5471 & 9 & 332.588 & -3.57 & 8.51361 & 78.5 & 1 \\
\hline Melatonin & 50.696 & 232.31 & 245.151 & 65.7819 & 4 & 0.3568 & 4 & 178.312 & -3.06 & 3.97496 & 39 & 0 \\
\hline meloxicam & 351.42 & 164.191 & 101.397 & 88.5629 & 7 & -0.1615 & 2 & 99.598 & -3.36 & 4.24029 & 61.1167 & 1 \\
\hline Melphalan & 305.23 & 164.736 & 101.153 & 77.5102 & 5 & 2.3515 & 8 & 66.56 & -3.92 & 7.34464 & 43.4556 & 1 \\
\hline meperidine & 247.37 & 161.874 & 101.98 & 72.4823 & 3 & 2.4651 & 4 & 29.543 & -2.35 & 4.14591 & 38.5833 & 0 \\
\hline Meptazinol & 23.466 & 233.39 & 255.586 & 71.9905 & 3 & 3.5125 & 2 & 200.37 & -2.82 & 3.848 & 33.5833 & 1 \\
\hline Mercaptopurine & 152.19 & 111.987 & 60.1268 & 41.4233 & 5 & 0.4524 & 0 & 57.366 & -2.19 & 1.64517 & 21.2667 & 0 \\
\hline Meropenem & 110.172 & 383.51 & 349.883 & 97.6798 & 9 & -2.0366 & 5 & 281.583 & -1.83 & 5.39707 & 67.4667 & 2 \\
\hline metaproterenol(orciprenaline) & 211.29 & 140.002 & 90.4087 & 57.8229 & 8 & 1.3864 & 4 & 72.711 & -1.48 & 4.22865 & 39.6667 & 3 \\
\hline Metergoline & 46.504 & 403.57 & 387.769 & 119.298 & 4 & 3.7523 & 5 & 305.493 & -4.93 & 5.29034 & 60.5 & 0 \\
\hline Metformin & 129.2 & 126.769 & 61.1555 & 35.8129 & 4 & 0.212 & 3 & 88.992 & -1.97 & 3.94527 & 25.8333 & 0 \\
\hline methadone & 309.49 & 180.576 & 115.096 & 97.3884 & 2 & 5.3821 & 7 & 20.309 & -4.72 & 5.72787 & 47.5833 & 0 \\
\hline Methapyrilene & 19.368 & 261.42 & 262.547 & 79.3882 & 2 & 2.2727 & 6 & 207.935 & -2.96 & 5.35926 & 33.4667 & 0 \\
\hline Methotrexate & 454.5 & 268.409 & 150.529 & 118.142 & 14 & 0.128201 & 9 & 210.548 & -3.42 & 8.87585 & 92 & 2 \\
\hline Methyldopa & 211.24 & 121.43 & 75.7615 & 53.2168 & 9 & 0.7861 & 3 & 103.778 & -1.97 & 3.20716 & 46.4167 & 3 \\
\hline Methylphenidate & 38.332 & 233.34 & 246.569 & 66.7274 & 4 & 2.1224 & 4 & 190.514 & -3.11 & 4.28636 & 37 & 0 \\
\hline Methylphenidate & 233.34 & 171.46 & 91.7685 & 66.7274 & 4 & 2.1224 & 4 & 38.332 & -3.11 & 4.28636 & 37 & 0 \\
\hline Methylprednisolone & 374.52 & 175.335 & 103.608 & 103.134 & 8 & 1.8404 & 2 & 94.826 & -3.54 & 4.12416 & 66.9167 & 3 \\
\hline Methysergide & 57.498 & 353.51 & 341.907 & 104.472 & 5 & 1.5005 & 4 & 268.582 & -3.2 & 4.56753 & 55.5 & 1 \\
\hline metoclopramide & 299.84 & 183.14 & 119.625 & 83.0188 & 5 & 1.2729 & 7 & 67.593 & -2.99 & 7.11817 & 44.6444 & 0 \\
\hline Metoclopramide & 203.26 & 128.905 & 68.4948 & 56.3486 & 2 & 1.6164 & 1 & 37.38 & -1.98 & 2.1955 & 37.75 & 0 \\
\hline Metolazone & 365.86 & 166.409 & 108.187 & 92.3093 & 5 & 2.1933 & 2 & 92.501 & -3.95 & 4.22722 & 60.1278 & 0 \\
\hline Metoprolol & 267.41 & 213.414 & 118.167 & 76.696 & 6 & 1.7894 & 9 & 50.723 & -2.82 & 7.66912 & 41.5 & 1 \\
\hline metronidazole & 171.18 & 110.966 & 67.0501 & 41.6633 & 5 & 0.3793 & 2 & 83.878 & -1.46 & 2.96884 & 37.3333 & 1 \\
\hline mexiletine & 179.29 & 233.878 & 146.94 & 54.9716 & 3 & 2.3508 & 3 & 35.257 & -2.52 & 3.4575 & 27.3333 & 0 \\
\hline Mianserin & 6.476 & 264.4 & 266.817 & 83.7778 & 1 & 3.5212 & 0 & 207.662 & -3.06 & 2.86486 & 36 & 0 \\
\hline mibefradil & 495.7 & 244.225 & 172.283 & 140.046 & 6 & 4.5625 & 12 & 67.46 & -5.68 & 8.99673 & 79.0833 & 0 \\
\hline Miconazole & 27.06 & 416.14 & 338.415 & 103.036 & 2 & 5.9949 & 6 & 294.312 & -7.27 & 6.73428 & 46.0778 & 0 \\
\hline midazolam & 325.79 & 290.75 & 208.399 & 88.2939 & 2 & 4.6312 & 1 & 30.19 & -4.52 & 3.82934 & 50.1444 & 0 \\
\hline mifobate & 358.67 & 164.125 & 103.989 & 76.7988 & 7 & 2.985 & 8 & 80.314 & -1.87 & 7.04263 & 54.0889 & 0 \\
\hline Minocycline & 457.53 & 218.613 & 131.737 & 121.052 & 13 & -2.5928 & 3 & 164.624 & -2.17 & 5.53286 & 91.5833 & 4 \\
\hline minoxidilne & 209.29 & 139.284 & 86.6577 & 59.8 & 4 & -2.0664 & 1 & 93.635 & -1.02 & 3.20992 & 36.5 & 0 \\
\hline mirtazapine & 265.39 & 170.272 & 104.43 & 81.9344 & 2 & 2.9079 & 0 & 19.368 & -2.38 & 3.05008 & 37 & 0 \\
\hline Misoprostol & 382.6 & 202.349 & 117.296 & 107.878 & 7 & 3.8945 & 15 & 83.832 & -4.37 & 11.0434 & 64.5833 & 2 \\
\hline Modafinil & 273.37 & 169.705 & 96.4589 & 77.3608 & 3 & 1.6196 & 5 & 60.165 & -2.64 & 4.60062 & 46.2556 & 0 \\
\hline montelukast & 586.231 & 288.272 & 196.549 & 169.725 & 6 & 8.2857 & 13 & 70.419 & -7.85 & 9.30437 & 85.7778 & 2 \\
\hline Morphine & 285.37 & 290.285 & 189.497 & 79.7595 & 6 & 1.3589 & 0 & 52.928 & -1.45 & 2.21228 & 45.25 & 2 \\
\hline Moxalactam(Latamoxef) & 520.52 & 235.485 & 138.46 & 124.703 & 16 & 0.095001 & 9 & 206.311 & -2.84 & 8.20128 & 103.05 & 3 \\
\hline Moxifloxacin & 401.48 & 215.076 & 125.48 & 108.075 & 7 & -1.993 & 4 & 83.803 & -2.71 & 4.63664 & 71.8333 & 1 \\
\hline moxonidine & 241.71 & 141.074 & 84.8712 & 62.666 & 6 & 1.3047 & 3 & 71.436 & -3.32 & 4.43187 & 34.1444 & 0 \\
\hline
\end{tabular}


Table 1 - (continuation)

\begin{tabular}{|c|c|c|c|c|c|c|c|c|c|c|c|c|}
\hline Name of drug & $\begin{array}{c}\text { Molecular } \\
\text { mass }\end{array}$ & $\begin{array}{c}\text { Molecular } \\
\text { surface } \\
\text { area }\end{array}$ & $\begin{array}{c}\text { Molecular } \\
\text { volume }\end{array}$ & $\begin{array}{l}\text { Molecular } \\
\text { refractivity }\end{array}$ & \begin{tabular}{|c|} 
Total $\mathrm{H}$ \\
bond \\
counting
\end{tabular} & $\log P$ & $\begin{array}{c}\text { Rotable } \\
\text { bonds }\end{array}$ & TPSA & $\log S$ & \begin{tabular}{|c|} 
Shape \\
flexibility \\
index
\end{tabular} & \begin{tabular}{|c|} 
Sum of \\
E-states \\
indices \\
\end{tabular} & $\begin{array}{l}\text { Hydroxyl } \\
\text { groups }\end{array}$ \\
\hline Nabumetone & 228.31 & 170.097 & 96.1728 & 68.4311 & 2 & 3.3705 & 4 & 26.305 & -5.07 & 3.54603 & 37.8333 & 0 \\
\hline N-Acetylprocainamide (Acecainide) & 277.41 & 199.346 & 112.044 & 80.1326 & 5 & 0.6397 & 7 & 61.434 & -3.04 & 7.07517 & 47.6667 & 0 \\
\hline nadolol & 309.45 & 170.56 & 113.879 & 85.5243 & 9 & 1.2691 & 6 & 81.945 & -2.14 & 5.57887 & 52.25 & 3 \\
\hline Nafcillin & 98.769 & 413.5 & 354.173 & 106.711 & 6 & 1.5601 & 4 & 293.204 & -4.4 & 4.98114 & 73.05 & 0 \\
\hline nalbuphine & 357.49 & 191.19 & 131.454 & 96.6362 & 8 & 1.7042 & 2 & 73.156 & -2.23 & 3.13426 & 55.5 & 3 \\
\hline nalmefene & 339.47 & 174.44 & 113.129 & 94.8462 & 6 & 2.1596 & 2 & 52.928 & -2.61 & 2.71475 & 51.3333 & 2 \\
\hline naloxone & 327.41 & 161.691 & 103.805 & 88.3591 & 7 & 1.7416 & 2 & 70.999 & -1.76 & 2.80209 & 56 & 2 \\
\hline Naltrexone & 341.44 & 189.611 & 104.851 & 91.1382 & 7 & 1.7109 & 2 & 69.999 & -2.05 & 2.68542 & 55.3333 & 2 \\
\hline naproxen & 454.53 & 224.899 & 139.571 & 115.272 & 8 & 2.419 & 7 & 46.533 & -4.4 & 6.1064 & 83.2167 & 1 \\
\hline Naratriptan & 335.51 & 213.881 & 124.714 & 94.5036 & 5 & 1.3125 & 5 & 65.198 & -3.47 & 4.86729 & 50.3167 & 0 \\
\hline Naringin & 225.065 & 580.59 & 452.397 & 133.73 & 22 & 0.093 & 6 & 400.011 & -2.16 & 8.84478 & 115.833 & 8 \\
\hline Nedocromil & 126.814 & 371.37 & 311.002 & 97.3645 & 9 & 0.264 & 5 & 248.495 & -3.91 & 4.98581 & 78.3333 & 2 \\
\hline nefazodone & 470.07 & 275.972 & 179.671 & 131.655 & 4 & 4.2872 & 10 & 55.451 & -3.74 & 8.95508 & 66.1444 & 0 \\
\hline nelfinavir & 567.861 & 271.287 & 196.161 & 161.832 & 9 & 4.6422 & 10 & 101.89 & -5.47 & 10.5979 & 87.55 & 2 \\
\hline Neomycin & 353.138 & 628.781 & 531.011 & 140.833 & 32 & -7.44 & 10 & 452.564 & -1.1 & 12.0567 & 119.833 & 7 \\
\hline netivudine & 282.28 & 174.264 & 107.215 & 65.4861 & 10 & -1.1366 & 4 & 63.58 & -1.87 & 4.29421 & 60.8333 & 3 \\
\hline Nevirapine & 266.33 & 179.177 & 99.4313 & 75.305 & 4 & 1.8723 & 1 & 63.58 & -3.41 & 2.86633 & 43.8333 & 0 \\
\hline nicardipine & 479.58 & 251.287 & 164.923 & 134.795 & 8 & 1.998 & 10 & 113.699 & -5.29 & 8.9118 & 88.3333 & 0 \\
\hline Nicotine & 162.26 & 130.329 & 69.9689 & 49.6227 & 2 & 1.4827 & 1 & 16.13 & -0.24 & 2.36077 & 22.5 & 0 \\
\hline nicotinic acid(niacin) & 123.12 & 90.7132 & 47.4626 & 30.628 & 4 & 0.898 & 1 & 50.191 & -0.17 & 1.85293 & 27.3333 & 1 \\
\hline nifedipine & 346.37 & 303.568 & 210.127 & 92.1635 & 7 & 0.2423 & 5 & 110.461 & -4.29 & 5.7028 & 70.1667 & 0 \\
\hline Nimodipine & 130.689 & 376.4 & 332.539 & 98.4379 & 9 & 0.0458 & 7 & 278.106 & -4.12 & 6.8307 & 77.1667 & 1 \\
\hline nisoldipine & 388.46 & 194.566 & 128.899 & 105.908 & 7 & 1.4562 & 7 & 110.461 & -4.83 & 7.08869 & 75 & 0 \\
\hline Nitrazepam & 281.29 & 156.601 & 89.3893 & 77.4351 & 5 & 2.1982 & 1 & 87.286 & -3.97 & 3.64352 & 56 & 0 \\
\hline nitrendipine & 360.4 & 184.162 & 122.533 & 96.9115 & 7 & 0.5848 & 6 & 110.461 & -4.41 & 6.27469 & 71.6667 & 0 \\
\hline Nitrofurantoin & 238.18 & 152.34 & 84.7954 & 53.1211 & 7 & 0.0407 & 3 & 120.735 & -2.76 & 3.24856 & 55.1667 & 0 \\
\hline Nizatidine & 331.5 & 205.386 & 115.333 & 89.1165 & 6 & 1.1397 & 10 & 86.008 & -3.93 & 9.29925 & 48.2667 & 0 \\
\hline Nordiazepam & 270.73 & 145.876 & 87.7843 & 74.9152 & 3 & 2.7626 & 1 & 41.462 & -4.09 & 3.41029 & 41.3111 & 0 \\
\hline Norepinephrinel & 86.707 & 169.2 & 176.178 & 43.8821 & 8 & 0.2225 & 2 & 117.054 & -1.13 & 2.77629 & 35.8333 & 3 \\
\hline Norfenefrine & 66.479 & 153.2 & 171.435 & 42.188 & 6 & 0.5069 & 2 & 113.434 & -0.91 & 2.59321 & 30.1667 & 2 \\
\hline Norfloxacin & 319.37 & 189.211 & 112.873 & 85.2424 & 6 & 0.846701 & 3 & 74.569 & -1.9 & 4.35954 & 61.6667 & 1 \\
\hline nortriptyline & 263.41 & 306.13 & 204.704 & 87.0364 & 2 & 4.1572 & 4 & 12.027 & -5.48 & 3.98206 & 36.8333 & 0 \\
\hline ofloxacin & 361.41 & 176.094 & 124.086 & 94.7015 & 6 & 0.848201 & 2 & 75.014 & -1.87 & 4.24503 & 67.6667 & 1 \\
\hline olanzapine & 312.47 & 185.213 & 118.151 & 93.1032 & 3 & 2.2728 & 1 & 35.159 & -3.52 & 3.90693 & 40.1333 & 0 \\
\hline Olsalazine & 139.782 & 302.26 & 274.043 & 77.282 & 12 & 3.1766 & 5 & 184.349 & -3.59 & 5.10618 & 69.3333 & 4 \\
\hline olsalazine & 302.26 & 160.489 & 98.4169 & 77.282 & 12 & 3.1766 & 5 & 139.782 & -3.59 & 5.10618 & 69.3333 & 4 \\
\hline omeprazole & 359.59 & 206.683 & 145.465 & 96.4368 & 9 & 0.137202 & 5 & 77.114 & -2.98 & 6.32132 & 48.0889 & 0 \\
\hline ondansetron & 293.4 & 175.488 & 111.212 & 87.2219 & 2 & 2.3371 & 2 & 39.831 & -3.07 & 3.31777 & 45.8333 & 0 \\
\hline oseltamivir & 312.46 & 183.362 & 124.71 & 84.2016 & 7 & 0.528102 & 8 & 90.66 & -2.66 & 7.8867 & 53.8333 & 0 \\
\hline Ouabain & 206.59 & 584.73 & 471.211 & 140.832 & 20 & -1.5009 & 4 & 426.734 & -2.1 & 7.31816 & 108 & 8 \\
\hline 0xacillin & 401.47 & 228.606 & 131.095 & 101.239 & 8 & 1.5823 & 4 & 112.738 & -3.67 & 4.83719 & 71.55 & 1 \\
\hline Oxalic_acid & 74.598 & 90.04 & 90.9757 & 14.4386 & 6 & -0.241 & 1 & 57.9416 & -0.14 & 1.43452 & 29.3333 & 2 \\
\hline oxatomide & 426.61 & 247.314 & 155.482 & 129.01 & 4 & 3.8589 & 7 & 61.69 & -4.04 & 6.4138 & 63.6667 & 0 \\
\hline Oxprenolol & 265.39 & 182.485 & 114.204 & 76.0002 & 6 & 2.2826 & 9 & 50.723 & -2.59 & 7.36471 & 43 & 1 \\
\hline oxybutynin & 252.29 & 144.977 & 81.098 & 71.5579 & 3 & 1.6598 & 0 & 63.403 & -3.2 & 2.73939 & 47.5 & 0 \\
\hline
\end{tabular}


RAJNISH KUMARA, ANJU SHARMA, PRITISH VARADWAJ, AUSAF AHMAD and GHULAM MD ASHRAF 189

Table 1 - (continuation).

\begin{tabular}{|c|c|c|c|c|c|c|c|c|c|c|c|c|}
\hline Name of drug & $\begin{array}{c}\text { Molecular } \\
\text { mass }\end{array}$ & $\begin{array}{c}\text { Molecular } \\
\text { surface } \\
\text { area }\end{array}$ & $\begin{array}{c}\text { Molecular } \\
\text { volume }\end{array}$ & $\begin{array}{l}\text { Molecular } \\
\text { refractivity }\end{array}$ & $\begin{array}{c}\text { Total } \mathrm{H} \\
\text { bond } \\
\text { counting }\end{array}$ & $\log P$ & $\begin{array}{l}\text { Rotable } \\
\text { bonds }\end{array}$ & TPSA & $\log S$ & $\begin{array}{c}\text { Shape } \\
\text { flexibility } \\
\text { index }\end{array}$ & $\begin{array}{l}\text { Sum of } \\
\text { E-states } \\
\text { indices }\end{array}$ & $\begin{array}{l}\text { Hydroxyl } \\
\text { groups }\end{array}$ \\
\hline oxyfedrine & 313.43 & 194.965 & 126.078 & 90.751 & 6 & 2.6061 & 8 & 58.56 & -4.16 & 6.46883 & 53.3333 & 1 \\
\hline Paclitaxel & 853.991 & 748.869 & 551.96 & 217.299 & 18 & 3.9632 & 14 & 221.307 & -5.19 & 12.3465 & 160.5 & 3 \\
\hline pafenolol & 337.52 & 229.341 & 150.191 & 95.7857 & 8 & 1.7211 & 10 & 82.614 & -3.58 & 9.74525 & 55 & 1 \\
\hline Pamidronate & 161.305 & 235.09 & 182.96 & 39.9314 & 14 & -1.1641 & 4 & 144.919 & -1.17 & 3.82633 & 52.8611 & 5 \\
\hline Papaverine & 49.828 & 339.42 & 330.046 & 95.7229 & 5 & 3.0613 & 6 & 260.052 & -4.42 & 5.71289 & 53.8333 & 0 \\
\hline Paromomycin & 347.34 & 615.73 & 503.565 & 134.243 & 32 & -6.8255 & 9 & 434.413 & -0.89 & 11.4718 & 120.333 & 8 \\
\hline pefloxacin & 333.4 & 187.613 & 120.99 & 89.3291 & 5 & 0.949701 & 3 & 65.7 & -1.96 & 4.59658 & 63.1667 & 1 \\
\hline Pentamidine & 118.218 & 340.47 & 333.214 & 98.7032 & 6 & 2.6629 & 10 & 272.485 & -4.16 & 8.2279 & 58.5 & 0 \\
\hline Pentazocine & 285.47 & 185.367 & 106.191 & 89.5115 & 3 & 4.2756 & 3 & 23.466 & -3.37 & 3.70644 & 40.5833 & 1 \\
\hline Pentoxifylline & 278.35 & 317.399 & 204.655 & 73.0241 & 4 & 0.315201 & 5 & 78.907 & -1.73 & 4.52343 & 52.3333 & 0 \\
\hline Phenacetin & 38.332 & 179.24 & 210.72 & 50.3514 & 3 & 0.9853 & 3 & 132.112 & 2 & 3.61004 & 31.5 & 0 \\
\hline phenglutarimide & 288.43 & 172.417 & 118.289 & 83.8526 & 4 & 1.788 & 6 & 49.407 & -3.08 & 5.64761 & 47.6667 & 0 \\
\hline phenobarbital & 232.26 & 132.267 & 76.1919 & 59.7463 & 5 & 1.5606 & 2 & 75.267 & -2.93 & 2.87015 & 47.4167 & 0 \\
\hline Phenoperidinl & 49.771 & 367.53 & 370.087 & 107.923 & 5 & 3.9213 & 8 & 292.125 & -3.98 & 6.77636 & 58.5833 & 1 \\
\hline Phenoxymethylpenicillin & 350.42 & 184.345 & 111.203 & 85.6734 & 7 & 0.570199 & 5 & 95.94 & -2.89 & 4.58454 & 63.05 & 1 \\
\hline Phenylbutazone & 308.41 & 206.886 & 115.7 & 88.7583 & 2 & 4.2216 & 5 & 40.618 & -3.33 & 4.6468 & 52.5 & 0 \\
\hline Phenylephrine & 167.23 & 139.62 & 74.809 & 46.9626 & 6 & 0.9152 & 3 & 52.483 & -0.88 & 3.22184 & 30.6667 & 2 \\
\hline Phenytoin & 252.29 & 170.463 & 96.6996 & 69.9813 & 4 & 2.2587 & 2 & 58.196 & -3.5 & 2.73939 & 46.9167 & 0 \\
\hline Physostigminel & 44.808 & 261.36 & 262.178 & 73.2575 & 4 & 1.2538 & 2 & 197.151 & -2.12 & 3.14236 & 41.3333 & 0 \\
\hline Pimozide & 479.781 & 273.84 & 178.089 & 132.674 & 3 & 4.3413 & 7 & 41.034 & -5.43 & 8.58552 & 70.3333 & 0 \\
\hline Pindolol & 248.36 & 155.823 & 84.9222 & 71.4608 & 6 & 1.5679 & 6 & 57.28 & -2.46 & 4.49301 & 39.1667 & 1 \\
\hline pirbuterol & 240.34 & 158.417 & 98.6598 & 64.6814 & 9 & 0.700001 & 5 & 85.603 & -1.59 & 4.95164 & 44.0833 & 3 \\
\hline Pirenzepine & 74.235 & 351.45 & 303.074 & 98.6471 & 6 & 0.5935 & 2 & 256.495 & 2.71 & 4.86874 & 59 & 0 \\
\hline piroxicam & 331.37 & 168.565 & 97.5722 & 84.9759 & 7 & -0.3903 & 2 & 99.599 & -3.37 & 4.07555 & 62.8167 & 1 \\
\hline piroximone & 217.25 & 134.997 & 79.007 & 59.6213 & 5 & -1.034 & 3 & 78.616 & -2.13 & 3.17317 & 41.8333 & 0 \\
\hline Practolol & 266.38 & 177.552 & 114.359 & 73.4565 & 7 & 0.6436 & 7 & 70.587 & -2.74 & 6.65708 & 46.1667 & 1 \\
\hline Pramipexole & 211.36 & 160.251 & 84.268 & 62.0095 & 4 & 1.2555 & 3 & 50.942 & -3.18 & 3.5818 & 25.9667 & 0 \\
\hline Pravastatin & 424.59 & 215.32 & 160.527 & 113.597 & 11 & 2.1716 & 11 & 124.288 & -3.24 & 9.37263 & 78.1667 & 4 \\
\hline praziquantel & 312.45 & 188.524 & 126.25 & 88.7847 & 2 & 2.1569 & 1 & 40.618 & -2.91 & 4.03538 & 48.8333 & 0 \\
\hline Prazosin & 383.45 & 230.534 & 129.731 & 103.279 & 7 & 1.163 & 4 & 106.962 & -2.74 & 5.82569 & 64.8333 & 0 \\
\hline Prednisolone & 360.49 & 180.896 & 107.283 & 98.5856 & 8 & 1.5099 & 2 & 94.826 & -3.18 & 3.89428 & 65.0833 & 3 \\
\hline Prednisone & 358.47 & 352.333 & 219.861 & 97.661 & 7 & 1.8777 & 2 & 91.669 & -3.51 & 3.73802 & 66.4167 & 2 \\
\hline Primidone & 218.28 & 151.442 & 77.2158 & 59.0376 & 4 & 1.4856 & 2 & 58.196 & -2.32 & 2.8511 & 40.25 & 0 \\
\hline Probenecid & 285.39 & 189.999 & 101.065 & 73.3121 & 5 & 2.3799 & 7 & 74.679 & -2.83 & 5.65245 & 52.3167 & 1 \\
\hline Procainamide & 235.37 & 179.215 & 99.6079 & 71.7508 & 4 & 1.0076 & 6 & 58.359 & -1.89 & 5.83063 & 38.5 & 0 \\
\hline Prochlorperazine & 11.41 & 373.98 & 349.604 & 109.909 & 2 & 3.5965 & 4 & 282.817 & -4.53 & 5.34115 & 42.7778 & 0 \\
\hline Progesterone & 314.51 & 168.243 & 112.93 & 92.7986 & 2 & 3.6889 & 1 & 34.142 & -4.76 & 3.41582 & 46.8333 & 0 \\
\hline Promethazine & 8.172 & 284.45 & 277.583 & 88.6067 & 1 & 3.6382 & 3 & 210.681 & -4.07 & 3.83254 & 36.1333 & 0 \\
\hline Propafenone & 58.56 & 341.49 & 363.571 & 100.206 & 6 & 3.4004 & 11 & 258.23 & -4.65 & 8.32312 & 56 & 1 \\
\hline propanolol & 259.38 & 172.039 & 108.396 & 76.8245 & 5 & 2.7969 & 6 & 41.489 & -3.51 & 4.90793 & 40.6667 & 1 \\
\hline propiverine & 367.53 & 201.466 & 132.8 & 107.525 & 4 & 3.982 & 8 & 38.777 & -4.76 & 6.77636 & 56.5833 & 0 \\
\hline Propoxyphene & 29.543 & 339.52 & 330.992 & 102.88 & 3 & 4.637 & 9 & 281.411 & -4.91 & 6.91435 & 52.5833 & 0 \\
\hline Propranolol & 259.38 & 172.039 & 108.396 & 76.8245 & 5 & 2.7969 & 6 & 41.489 & -3.51 & 4.90793 & 40.6667 & 1 \\
\hline Propylthiouracil & 168.23 & 119.861 & 73.0952 & 47.7039 & 4 & 1.3709 & 2 & 48.653 & -2.27 & 3.3177 & 26.2667 & 0 \\
\hline Proquazone & 34.897 & 278.38 & 276.9 & 84.6536 & 2 & 4.5173 & 2 & 206.557 & -4.48 & 3.68815 & 45.3333 & 0 \\
\hline
\end{tabular}


CLASSIFICATION OF ORAL BIOAVAILABILITY OF DRUGS BY MACHINE LEARNING APPROACHES

Table 1 - (continuation)

\begin{tabular}{|c|c|c|c|c|c|c|c|c|c|c|c|c|}
\hline Name of drug & $\begin{array}{c}\text { Molecular } \\
\text { mass }\end{array}$ & $\begin{array}{c}\text { Molecular } \\
\text { surface } \\
\text { area }\end{array}$ & $\begin{array}{c}\text { Molecular } \\
\text { volume }\end{array}$ & $\begin{array}{l}\text { Molecular } \\
\text { refractivity }\end{array}$ & $\begin{array}{c}\text { Total } \mathrm{H} \\
\text { bond } \\
\text { counting }\end{array}$ & $\log P$ & $\begin{array}{c}\text { Rotable } \\
\text { bonds }\end{array}$ & TPSA & $\log S$ & $\begin{array}{c}\text { Shape } \\
\text { flexibility } \\
\text { index }\end{array}$ & $\begin{array}{l}\text { Sum of } \\
\text { E-states } \\
\text { indices }\end{array}$ & $\begin{array}{l}\text { Hydroxyl } \\
\text { groups }\end{array}$ \\
\hline Proscillaridin & 129.591 & 530.72 & 453.708 & 140.053 & 12 & 2.6956 & 3 & 397.732 & -4.09 & 6.58443 & 88.25 & 4 \\
\hline Protriptyline & 263.41 & 179.399 & 100.471 & 87.303 & 2 & 4.3343 & 4 & 12.027 & -6.06 & 3.98206 & 37 & 0 \\
\hline Pseudoephedrine & 165.26 & 135.097 & 72.5887 & 49.6867 & 4 & 1.6127 & 3 & 32.255 & -1.3 & 3.25867 & 26.8333 & 1 \\
\hline Quetiapine & 383.55 & 229.572 & 133.912 & 111.573 & 5 & 2.8402 & 6 & 48.83 & -3.98 & 6.53087 & 53.4667 & 1 \\
\hline Quinidine & 324.46 & 210.108 & 130.596 & 94.6611 & 5 & 3.0841 & 4 & 45.592 & -2.99 & 4.37891 & 49.5 & 1 \\
\hline Quinine & 324.46 & 211.193 & 118.3 & 94.6611 & 5 & 3.0841 & 4 & 45.592 & -2.99 & 4.37891 & 49.5 & 1 \\
\hline Raffinose & 268.678 & 504.5 & 399.853 & 101.187 & 27 & -3.6862 & 8 & 341.611 & 0.12 & 9.18478 & 108.083 & 11 \\
\hline Raloxifene & 69.999 & 473.62 & 427.153 & 136.943 & 7 & 4.89 & 7 & 344.646 & -5.97 & 6.94474 & 74.3 & 2 \\
\hline ranitidine & 314.45 & 199.237 & 129.643 & 85.127 & 6 & 0.715301 & 10 & 86.256 & -3.6 & 8.4307 & 50.1333 & 0 \\
\hline recainam & 263.43 & 174.833 & 114.727 & 79.552 & 5 & 2.2306 & 6 & 53.15 & 3.43 & 6.70032 & 41 & 0 \\
\hline repaglinide & 452.65 & 221.416 & 156.016 & 130.608 & 6 & 4.7464 & 10 & 78.869 & -5.19 & 9.32307 & 74.3333 & 1 \\
\hline reproterol & 389.46 & 211.392 & 144.334 & 100.886 & 11 & -0.098 & 7 & 134.547 & -2.13 & 6.41085 & 74.5 & 3 \\
\hline ribavirin & 244.24 & 128.405 & 85.8159 & 54.3191 & 11 & -2.1039 & 3 & 143.73 & -0.87 & 3.86949 & 52.6667 & 3 \\
\hline rimiterol & 223.3 & 142.038 & 91.31 & 60.4681 & 8 & 1.4233 & 2 & 72.711 & -1.51 & 3.55505 & 40.1667 & 3 \\
\hline Risperidone & 410.54 & 237.698 & 139.642 & 113.851 & 5 & 2.2349 & 4 & 64.167 & -3.47 & 5.87601 & 66.1667 & 0 \\
\hline Ritodrine & 72.711 & 287.39 & 297.466 & 82.4421 & 8 & 3.0722 & 6 & 224.023 & -3.21 & 5.47116 & 50.8333 & 3 \\
\hline Rizatriptan & 269.39 & 197.087 & 107.708 & 82.8446 & 4 & 1.6758 & 5 & 49.747 & -2.9 & 4.30232 & 39.6667 & 0 \\
\hline rofecoxib & 314.37 & 156.382 & 96.1324 & 83.8311 & 4 & 2.2409 & 3 & 60.447 & -4.47 & 3.68081 & 56.3167 & 0 \\
\hline Ropinirole & 260.42 & 186.068 & 104.894 & 79.5707 & 3 & 2.2802 & 7 & 32.336 & -2.87 & 5.1103 & 38.6667 & 0 \\
\hline rosiglitazone & 357.46 & 205.323 & 130.929 & 97.0371 & 5 & 3.1267 & 7 & 71.533 & -3.97 & 6.26016 & 57.8 & 0 \\
\hline saccharin & 183.19 & 101.349 & 54.9342 & 41.8214 & 4 & 0.2558 & 0 & 63.24 & -1.45 & 1.33293 & 36.8167 & 0 \\
\hline Salicylic acid & 138.13 & 98.4897 & 52.0031 & 34.5105 & 5 & 1.4606 & 1 & 57.527 & -1.09 & 1.79792 & 32 & 2 \\
\hline Saquinavir & 166.746 & 670.941 & 612.855 & 186.173 & 12 & 2.9936 & 13 & 524.271 & -5.43 & 12.5189 & 114.583 & 1 \\
\hline Scopolamine & 303.39 & 191.134 & 121.111 & 79.7213 & 6 & 1.0072 & 5 & 62.299 & -1.66 & 3.5316 & 49.8333 & 1 \\
\hline Selegiline & 187.31 & 220.66 & 164.316 & 61.3537 & 1 & 2.8721 & 5 & 3.238 & -3.87 & 4.22382 & 28.5 & 0 \\
\hline sildenafil & 473.63 & 242.726 & 168.173 & 126.15 & 8 & 2.1254 & 7 & 113.432 & -2.68 & 7.66379 & 77.65 & 0 \\
\hline Simvastatin & 418.63 & 400.76 & 337.381 & 117.682 & 6 & 4.4326 & 7 & 72.838 & -4.53 & 7.36399 & 67.5833 & 1 \\
\hline Sodium_oxybate & 57.527 & 104.12 & 125.317 & 23.7999 & 5 & -0.2905 & 3 & 81.1842 & 0.68 & 3.54814 & 25.1667 & 2 \\
\hline Sorivudine & 348.15 & 171.478 & 116.56 & 69.7954 & 10 & 0.1478 & 4 & 124.785 & -1.04 & 5.00317 & 59.3958 & 3 \\
\hline Sotalol & 272.4 & 161.101 & 102.727 & 71.4893 & 7 & 0.721401 & 6 & 78.424 & -2.54 & 5.27559 & 46.8167 & 1 \\
\hline spironolactone & 416.62 & 193.513 & 135.012 & 113.645 & 4 & 2.3927 & 2 & 60.447 & -5.32 & 4.49946 & 62.3833 & 0 \\
\hline Stavudine & 224.24 & 217.19 & 154.9 & 57.5 & 6 & 0.1812 & 2 & 84.329 & -0.46 & 3.27 & 42.66 & 1 \\
\hline Streptomycin & 331.434 & 581.67 & 478.436 & 128.64 & 27 & -4.5666 & 11 & 409.082 & -1.66 & 11.4848 & 119.25 & 7 \\
\hline Streptozocin & 151.917 & 265.26 & 233.621 & 55.9552 & 13 & -1.7592 & 2 & 182.959 & -0.9 & 5.60039 & 60.8333 & 4 \\
\hline sudoxicam & 337.39 & 156.014 & 93.4781 & 83.4138 & 7 & -0.176 & 2 & 99.599 & -3.12 & 4.00206 & 59.45 & 1 \\
\hline Sulfadiazine & 250.3 & 160.981 & 88.6155 & 64.1694 & 6 & 0.7233 & 3 & 97.976 & -2.62 & 3.71719 & 45.8167 & 0 \\
\hline Sulfamethoxazole & 253.3 & 165.181 & 89.1154 & 64.4952 & 6 & 0.9292 & 3 & 98.224 & -2.47 & 3.34749 & 45.9833 & 0 \\
\hline sulfasalazine & 398.42 & 202.315 & 120.273 & 103.787 & 11 & 4.0621 & 7 & 141.316 & -3.93 & 6.5744 & 78.4833 & 2 \\
\hline Sulfinpyrazone & 404.51 & 216.172 & 125.145 & 113.56 & 3 & 3.5477 & 6 & 57.689 & -3.1 & 5.7573 & 68.0889 & 0 \\
\hline Sulfisoxazole & 267.33 & 161.668 & 90.7885 & 68.2267 & 6 & 1.2754 & 3 & 98.224 & -2.93 & 3.58556 & 47.65 & 0 \\
\hline sulindac & 356.43 & 174.271 & 113.339 & 99.4754 & 4 & 2.6837 & 5 & 54.37 & -4.15 & 4.78972 & 64.9222 & 1 \\
\hline Sulpiride & 341.47 & 154.275 & 98.1515 & 88.1339 & 7 & 0.111501 & 6 & 101.735 & -2.8 & 5.87737 & 58.8167 & 0 \\
\hline sultopride & 354.51 & 186.724 & 124.514 & 94.4786 & 6 & 1.0793 & 7 & 75.712 & -3.25 & 6.50956 & 58.3167 & 0 \\
\hline sumatriptan & 295.44 & 186.336 & 113.896 & 82.3314 & 5 & 0.819501 & 6 & 72.838 & -3.37 & 4.59358 & 46.4833 & 0 \\
\hline Tacrine & 38.915 & 198.29 & 215.179 & 61.9435 & 2 & 2.8303 & 0 & 139.605 & -3.16 & 2.17479 & 29.3333 & 0 \\
\hline
\end{tabular}


RAJNISH KUMARA, ANJU SHARMA, PRITISH VARADWAJ, AUSAF AHMAD and GHULAM MD ASHRAF 191

Table 1 - (continuation).

\begin{tabular}{|c|c|c|c|c|c|c|c|c|c|c|c|c|}
\hline Name of drug & $\begin{array}{c}\text { Molecular } \\
\text { mass }\end{array}$ & $\begin{array}{c}\text { Molecular } \\
\text { surface } \\
\text { area }\end{array}$ & $\begin{array}{c}\text { Molecular } \\
\text { volume }\end{array}$ & $\begin{array}{l}\text { Molecular } \\
\text { refractivity }\end{array}$ & $\begin{array}{c}\text { Total } \mathrm{H} \\
\text { bond } \\
\text { counting }\end{array}$ & $\log P$ & $\begin{array}{l}\text { Rotable } \\
\text { bonds }\end{array}$ & TPSA & $\log S$ & $\begin{array}{c}\text { Shape } \\
\text { flexibility } \\
\text { index }\end{array}$ & $\begin{array}{l}\text { Sum of } \\
\text { E-states } \\
\text { indices }\end{array}$ & $\begin{array}{l}\text { Hydroxyl } \\
\text { groups }\end{array}$ \\
\hline Tacrolimus & 178.376 & 804.141 & 712.467 & 215.711 & 15 & 5.1656 & 8 & 630.309 & -5.3 & 17.5439 & 137.083 & 3 \\
\hline Tamsulosin & 408.56 & 247.282 & 142.088 & 108.863 & 8 & 2.2098 & 11 & 99.894 & -4.79 & 8.55585 & 66.9833 & 0 \\
\hline telmisartan & 514.67 & 281.189 & 188.248 & 153.819 & 5 & 7.5316 & 7 & 72.951 & -5.17 & 7.03226 & 83.1667 & 1 \\
\hline Temazepam & 300.76 & 169.913 & 99.8883 & 81.0103 & 4 & 3.0462 & 1 & 52.901 & -3.75 & 3.85211 & 48.6444 & 1 \\
\hline Tenidap & 320.76 & 158.628 & 96.5863 & 81.3625 & 5 & 1.0015 & 2 & 85.327 & -4.53 & 3.56139 & 53.2778 & 1 \\
\hline Tenofovir & 136.394 & 287.25 & 269.268 & 66.806 & 10 & 0.2327 & 5 & 186.631 & -1.57 & 4.99343 & 53.1389 & 2 \\
\hline tenoxicam & 337.39 & 158.438 & 92.6938 & 83.7786 & 7 & -0.6337 & 2 & 99.598 & -3.12 & 4.00206 & 59.45 & 1 \\
\hline Terazosin & 387.49 & 251.764 & 146.206 & 104.455 & 7 & 0.545001 & 4 & 103.056 & -2.41 & 6.20721 & 63 & 0 \\
\hline terbutaline & 225.32 & 143.831 & 90.9709 & 62.4605 & 8 & 1.4646 & 4 & 72.711 & -1.59 & 4.04284 & 41.5833 & 3 \\
\hline Terfenadine & 43.694 & 471.74 & 493.619 & 146.065 & 5 & 7.0287 & 9 & 389.271 & -6.01 & 8.10866 & 70.3333 & 2 \\
\hline Terguride & 51.365 & 340.52 & 340.066 & 101.191 & 4 & 1.9414 & 3 & 259.828 & -3.59 & 4.49784 & 49.8333 & 0 \\
\hline Testosterone & 288.47 & 161.401 & 110.73 & 84.5227 & 3 & 2.8985 & 0 & 37.299 & -3.94 & 2.95854 & 42.1667 & 1 \\
\hline Tetrabenazine & 38.777 & 317.47 & 332.937 & 91.307 & 4 & 3.3272 & 4 & 247.83 & -2.94 & 4.85273 & 47.8333 & 0 \\
\hline tetracycline & 444.48 & 186.194 & 130.862 & 113.42 & 15 & -3.1477 & 2 & 181.614 & -2.52 & 4.92084 & 93.6667 & 5 \\
\hline theophylline & 184.23 & 116.621 & 76.3016 & 43.6117 & 6 & -0.8989 & 0 & 72.693 & -0.9 & 1.91801 & 34.5 & 0 \\
\hline Tiagabine & 375.58 & 215.249 & 126.348 & 110.029 & 4 & 3.8023 & 7 & 40.537 & -4.25 & 6.44438 & 50.6 & 1 \\
\hline Ticarcillin & 135.649 & 383.44 & 320.932 & 88.1638 & 8 & -0.3761 & 3 & 272.509 & -2.89 & 4.64702 & 72.35 & 0 \\
\hline Timolol & 316.47 & 199.844 & 131.128 & 85.235 & 8 & 0.8882 & 7 & 79.745 & -3.07 & 6.91271 & 45.05 & 1 \\
\hline Tizanidine & 62.202 & 253.73 & 219.862 & 65.0233 & 5 & 2.2647 & 2 & 160.752 & -2.92 & 3.44448 & 31.2778 & 0 \\
\hline tobramycin & 467.6 & 230.856 & 172.331 & 106.695 & 24 & -5.5567 & 6 & 268.191 & -0.94 & 8.78724 & 88.6667 & 5 \\
\hline Tocainide & 192.29 & 154.206 & 81.1827 & 57.0756 & 4 & 1.4643 & 2 & 55.121 & -2.08 & 3.38373 & 33.5 & 0 \\
\hline tolbutamide & 270.38 & 171.713 & 103.6 & 70.2696 & 5 & 2.2143 & 5 & 75.267 & -0.13 & 5.45695 & 47.8167 & 0 \\
\hline Tolcapone & 273.26 & 154.84 & 94.1415 & 72.3876 & 7 & 3.12 & 2 & 103.351 & -3.68 & 3.71027 & 60.6667 & 2 \\
\hline tolmesoxide & 214.3 & 130.325 & 82.6951 & 58.3038 & 3 & 0.962301 & 3 & 35.539 & -1.72 & 3.80157 & 33.0889 & 0 \\
\hline Tolterodine & 325.54 & 200.988 & 117.517 & 103.668 & 3 & 5.8232 & 7 & 23.466 & -4.78 & 6.58654 & 47.6667 & 1 \\
\hline Topotecan & 421.49 & 228.368 & 137.731 & 114.729 & 9 & 0.160301 & 3 & 104.896 & -2.37 & 4.69548 & 76.0833 & 2 \\
\hline torasemide & 348.46 & 187.806 & 115.067 & 91.5648 & 7 & 2.0278 & 5 & 100.186 & -3.77 & 6.12097 & 61.4833 & 0 \\
\hline toremifene & 406 & 217.154 & 145.697 & 124.234 & 2 & 5.9097 & 10 & 12.474 & -6 & 8.06225 & 54.8111 & 0 \\
\hline tramadol & 263.42 & 166.376 & 108.113 & 78.2688 & 4 & 2.3021 & 4 & 32.7 & -2.55 & 4.6085 & 38.9167 & 1 \\
\hline Trandolapril & 95.94 & 430.6 & 414.857 & 115.785 & 8 & 3.2778 & 10 & 337.036 & -4.32 & 8.48701 & 74.3333 & 1 \\
\hline Tranexamic acid & 157.24 & 113.738 & 75.6231 & 41.9035 & 5 & 0.6158 & 2 & 63.322 & -0.94 & 3.02492 & 28.8333 & 1 \\
\hline trapidil & 205.3 & 136.895 & 84.9866 & 61.2336 & 3 & 1.5741 & 3 & 46.329 & -2.03 & 3.6371 & 31 & 0 \\
\hline trazodone & 371.91 & 225.257 & 140.725 & 105.159 & 3 & 2.7936 & 5 & 45.788 & -2.43 & 5.57443 & 52.4778 & 0 \\
\hline TRH & 150.282 & 362.44 & 339.504 & 89.5049 & 9 & -3.0287 & 6 & 269.923 & -2.46 & 6.10122 & 69.8333 & 0 \\
\hline Triamcinolone_acetonide & 93.066 & 420.52 & 342.225 & 107.284 & 8 & 1.4153 & 2 & 314.749 & -3.72 & 4.16041 & 77.1667 & 2 \\
\hline triamterene & 253.3 & 149.819 & 83.0386 & 75.1259 & 7 & 1.3677 & 1 & 129.637 & -2.42 & 3.62249 & 45.6667 & 0 \\
\hline Triazolam & 343.23 & 180.427 & 107.47 & 92.7297 & 3 & 4.8512 & 1 & 43.082 & -4.27 & 4.2637 & 44.4556 & 0 \\
\hline Trimethoprim & 290.36 & 195.359 & 109.67 & 81.5094 & 7 & 1.0485 & 5 & 105.532 & -2.67 & 5.61871 & 49.6667 & 0 \\
\hline Trovafloxacin & 416.39 & 191.532 & 129.786 & 101.388 & 7 & 0.5559 & 3 & 101.457 & -3.53 & 4.3726 & 88.6667 & 1 \\
\hline urapidil & 387.54 & 237.156 & 156.811 & 108.734 & 5 & 0.830701 & 7 & 71.4747 & -2.64 & 7.05848 & 62.8333 & 0 \\
\hline Uridine & 125.043 & 244.23 & 215.726 & 52.8005 & 11 & -0.6807 & 2 & 169.119 & -0.66 & 3.62125 & 53.6667 & 4 \\
\hline valacyclovir & 323.38 & 190.553 & 126.981 & 77.9553 & 10 & 0.045301 & 8 & 151.165 & -2 & 6.74278 & 61.5 & 0 \\
\hline valproic acid & 144.24 & 108.314 & 69.3015 & 40.2491 & 3 & 2.6079 & 5 & 37.299 & -1.79 & 5.21715 & 26 & 1 \\
\hline Valsartan & 435.58 & 390.46 & 331.32 & 125.791 & 8 & 4.9235 & 10 & 112.075 & -4.28 & 9.16053 & 75.8333 & 1 \\
\hline Venlafaxine & 277.45 & 295.93 & 230.19 & 83.0234 & 4 & 2.5538 & 5 & 32.7 & -3.08 & 5.19071 & 40.4167 & 1 \\
\hline
\end{tabular}


Table 1 - (continuation)

\begin{tabular}{|l|c|c|c|c|c|c|c|c|c|c|c|c|}
\hline Name of drug & $\begin{array}{c}\text { Molecular } \\
\text { mass }\end{array}$ & $\begin{array}{c}\text { Molecular } \\
\text { surface } \\
\text { area }\end{array}$ & $\begin{array}{c}\text { Molecular } \\
\text { volume }\end{array}$ & $\begin{array}{c}\text { Molecular } \\
\text { refractivity }\end{array}$ & $\begin{array}{c}\text { Total } \mathrm{H} \\
\text { bond } \\
\text { counting }\end{array}$ & logP & $\begin{array}{c}\text { Rotable } \\
\text { bonds }\end{array}$ & TPSA & logS & $\begin{array}{c}\text { Shape } \\
\text { flexibility } \\
\text { index }\end{array}$ & $\begin{array}{c}\text { Sum of } \\
\text { E-states } \\
\text { indices }\end{array}$ & $\begin{array}{c}\text { Hydroxyl } \\
\text { groups }\end{array}$ \\
\hline Vidarabine & 139.551 & 267.28 & 231.946 & 63.2276 & 11 & -1.2405 & 2 & 173.449 & -1.14 & 3.7758 & 52.3333 & 3 \\
Vigabatrin & 129.18 & 102.564 & 62.2648 & 34.2903 & 5 & 0.1732 & 4 & 63.322 & -0.13 & 3.91763 & 28 & 1 \\
Viloxazine & 237.33 & 165.269 & 101.612 & 65.2015 & 5 & 1.2294 & 5 & 39.79 & -2.28 & 4.9416 & 35.1667 & 0 \\
Vincamine & 53.009 & 356.51 & 314.281 & 99.2316 & 5 & 3.3193 & 3 & 265.855 & -2.57 & 3.64318 & 54.5 & 1 \\
Vinpocetine & 34.477 & 348.48 & 323.043 & 104.33 & 2 & 2.961 & 4 & 262.052 & -4.34 & 3.57611 & 52.5833 & 0 \\
Viomycin & 685.811 & 352.709 & 250.522 & 161.163 & 28 & -9.204 & 11 & 392.852 & -2.44 & 17.4738 & 144.333 & 3 \\
Warfarin & 322.49 & 199.444 & 126.659 & 87.2306 & 5 & 3.1228 & 4 & 67.51 & -3.32 & 5.52599 & 51.8333 & 1 \\
Xamoterol & 103.287 & 339.44 & 349.101 & 87.8404 & 10 & -0.3301 & 8 & 250.214 & -1.99 & 8.26306 & 59.3333 & 2 \\
Ximoprofen & 261.35 & 158.982 & 103.222 & 72.308 & 6 & 3.9265 & 4 & 69.891 & -3.66 & 4.78079 & 47.3333 & 2 \\
Zalcitabine & 211.25 & 142.706 & 87.523 & 52.4398 & 6 & -0.3252 & 2 & 93.281 & -0.34 & 3.20992 & 40 & 1 \\
Zanamivir & 332.36 & 299.62 & 236.33 & 75.799 & 15 & -3.1695 & 7 & 198.217 & -2.35 & 7.44706 & 74.3333 & 4 \\
Zidovudine & 266.27 & 166.922 & 108.439 & 63.8679 & 7 & 0.4937 & 3 & 133.812 & -0.68 & 4.74792 & 56.5 & 1 \\
Zimeldine & 16.13 & 317.25 & 279.249 & 84.7307 & 2 & 3.7813 & 5 & 213.104 & -4.12 & 5.23579 & 36.2292 & 0 \\
Ziprasidone & 412.97 & 219.951 & 145.71 & 116.185 & 4 & 2.9721 & 4 & 48.466 & -4.76 & 5.2439 & 54.2778 & 0 \\
Zolmitriptan & 287.4 & 192.225 & 110.293 & 82.4384 & 5 & 1.672 & 5 & 57.361 & -3.18 & 4.16605 & 45.1667 & 0 \\
Zolpidem & 321.57 & 229.887 & 147.032 & 94.2383 & 4 & 2.5813 & 3 & 37.616 & -0.99 & 5.44004 & 43.1667 & 0 \\
Zotepine & 12.472 & 331.88 & 312.396 & 97.3374 & 2 & 3.4929 & 4 & 252.163 & -5.63 & 4.88846 & 40.4445 & 0 \\
\hline
\end{tabular}

Table 2 - Detailed result of three classifiers. TP $=$ number of true positives, TN $=$ number of true negatives, FP $=$ number of false positives, $\mathrm{FN}=$ number of false negatives, $\mathrm{YI}=$ Youden's Index, $\mathrm{AUC}=$ area under curve.

\begin{tabular}{c|c|c|c|c|c|c|c|c|c|c}
\hline Classifier & TP & TN & FP & FN & YI & AUC & Sensitivity & Specificity & Accuracy & Precision \\
\hline ANN & 87 & 34 & 3 & 4 & 0.87 & 0.958 & $95.60 \%$ & $91.89 \%$ & $94.53 \%$ & $94.53 \%$ \\
Bayesian & 84 & 34 & 3 & 7 & 0.84 & 0.967 & $92.30 \%$ & $91.89 \%$ & $92.19 \%$ & $92.19 \%$ \\
SVM & 87 & 36 & 1 & 4 & 0.93 & 0.965 & $95.60 \%$ & $97.30 \%$ & $96.85 \%$ & $98.86 \%$ \\
\hline
\end{tabular}

The generated model was then tested with test dataset to examine the accuracy of model.

\subsubsection{Classification by Artificial Neural Network}

MATLAB nntool box was used for implementation of ANN. Classification was carried out using feed-forward back propagation. Mean square error and tan sigmoid was used as performance and transfer function respectively. Several neural network architectures were constructed using different combinations of hidden layers and number of neurons in the hidden layers. Among all the generated networks, one having the best efficiency was chosen as final network. It consists of an input layer, one hidden layer having 10 neurons and an output layer. The weights and bias values were fixed and the network was then tested using a test set of 127 drugs. Confusion matrix was constructed to calculate accuracy of network.

\subsubsection{Classification by Bayesian classifier}

WEKA was used to implement Bayesian classification. The training and test datasets were the same as used in above two classifiers. Both the test set and training set were converted into WEKA format (.arff file) using a small perl script. Bayes network learns using various search algorithms and quality measures. Base class function provided network structure, conditional probability distributions etc and therefore facilitates common to Bayes Network learning algorithm. Genetic algorithm is used as search algorithm during the classification.

\section{RESULTS AND DISCUSSION}

The prediction accuracy of SVM classifier for the test set came out to be $96.85 \%$. Classifier efficiency was determined by calculating number of true positives (TP), number of true negatives (TN), number of false positives (FP) and number of false neg- 


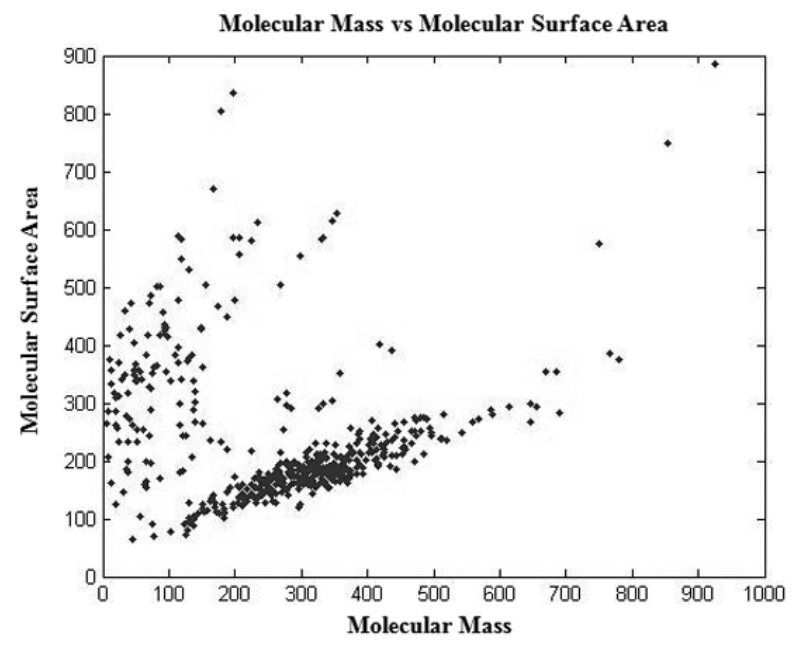

(a)

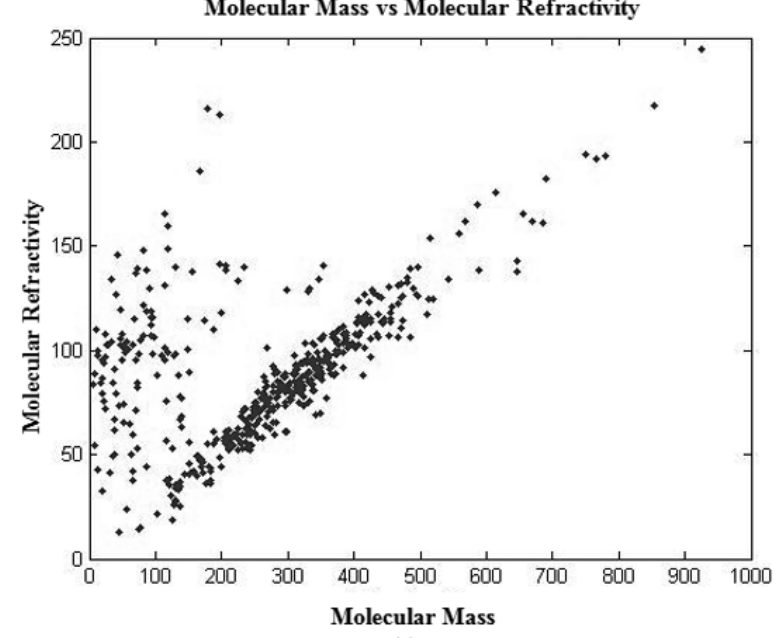

(c)

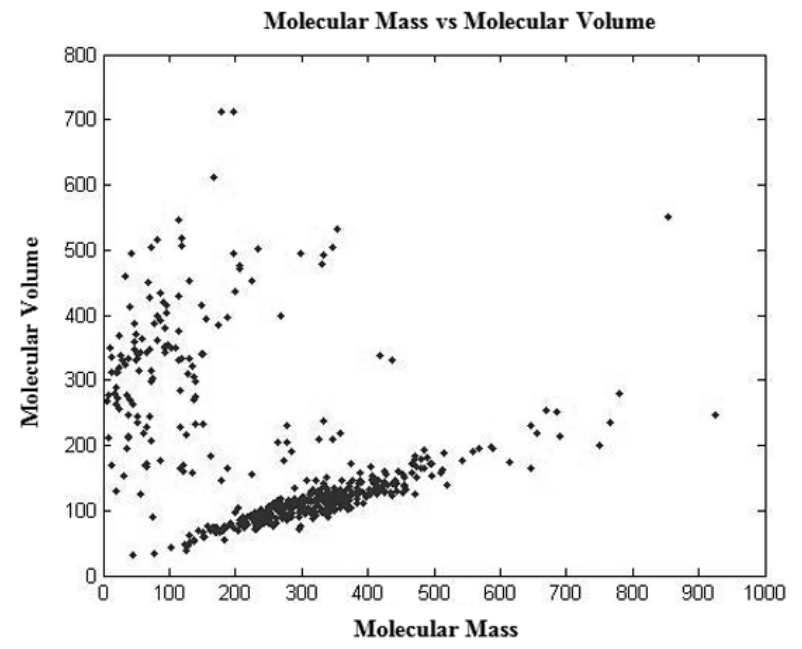

(b)

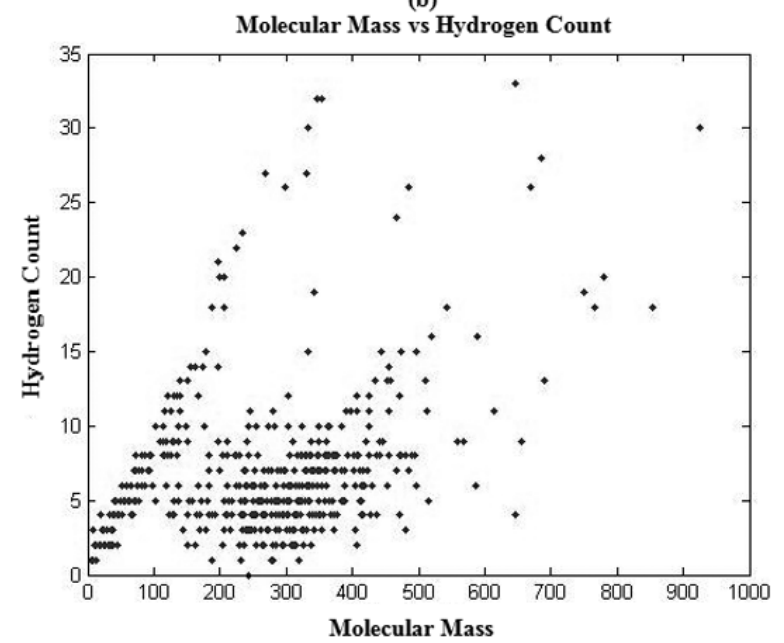

(d)

Figure 1 - Plot between different descriptors. (a): Molecular Surface Area vs. Molecular Mass, (b): Molecular Volume vs. Molecular Mass, (c): Molecular Refractivity vs. Molecular Mass, (d): Hydrogen Count vs. Molecular mass.

atives (FN) and these values were found to be $87,36,1$, and 4 respectively. Using these variables, Sensitivity came out to be $95.6 \%$ and $0.79 \%$ false positive proportion. Specificity was found to be $97.30 \%$ with $3.15 \%$ false negative proportion. Youden's Index was calculated as 0.929 . Area under ROC was found to be 0.965 .

From confusion matrix of ANN Number of TP, TN, FP and FN were calculates as $87,34,3,4$ respectively. Sensitivity and Specificity of the network was found to be $95.60 \%$ and $91.89 \%$. Overall accuracy of network is 94.53 . Youden's Index was calculated as 0.87 . Area under ROC curve was 0.958 .

The accuracy of prediction for Bayesian classifier was found 92.19 for test set. Total correctly classified instances were found to be 118 while incorrectly classified instances were 10 . The number of TP, TN, FP and FN were found to be 84, 34, 3 and
7 respectively. The confusion matrix for the prediction was generated. F-test result was 0.923. Area under ROC curve was found to be 0.967 , whereas Youden's index was calculated as 0.84 . Quite a few researchers have been tried to generate absorption models using different machine learning approaches and reported good results $[6,24]$. This is the first time we are presenting a comparative study between three potential machine learning approaches viz. SVM, ANN and Bayesian for the same dataset. The basic idea behind generating the three models is to compare these models and to get some idea about the most efficient machine learning approach for considered oral bioavailability dataset.

The performance of developed classifiers using SVM, ANN and Bayesian classifier were compared to determine most efficient classifier among three for prediction of oral bioavailabil- 


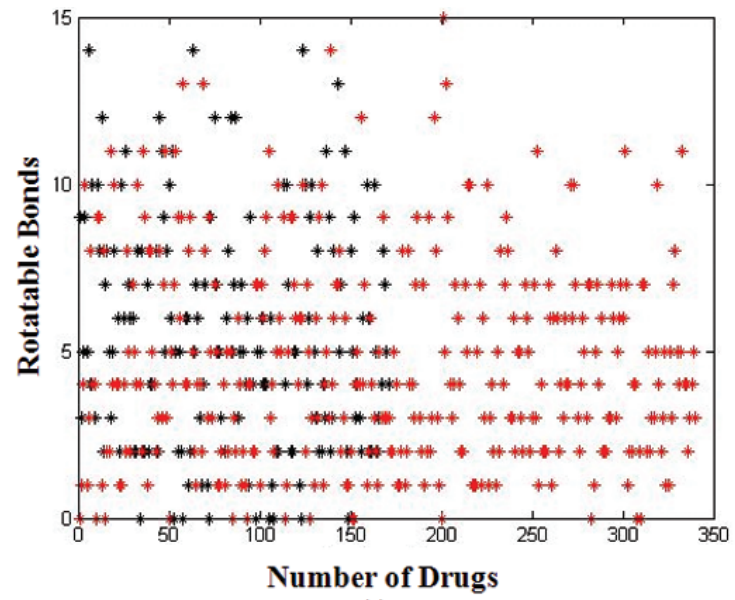

(a)

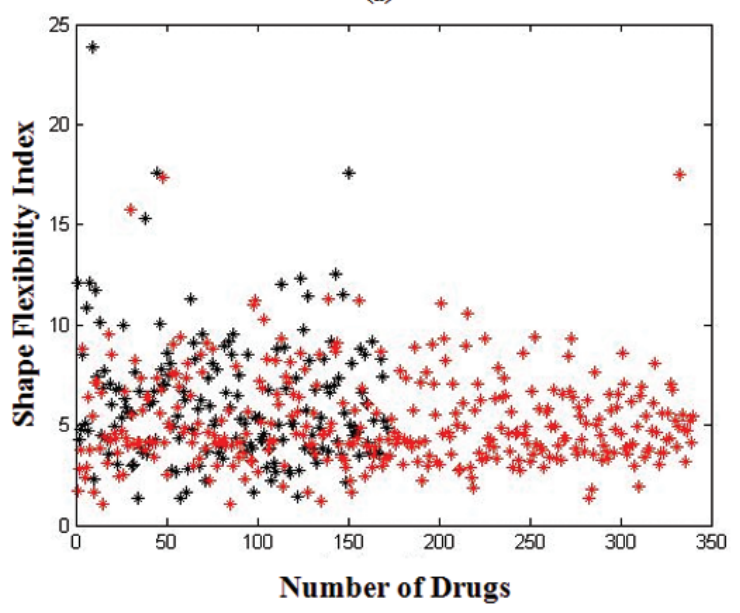

(c)

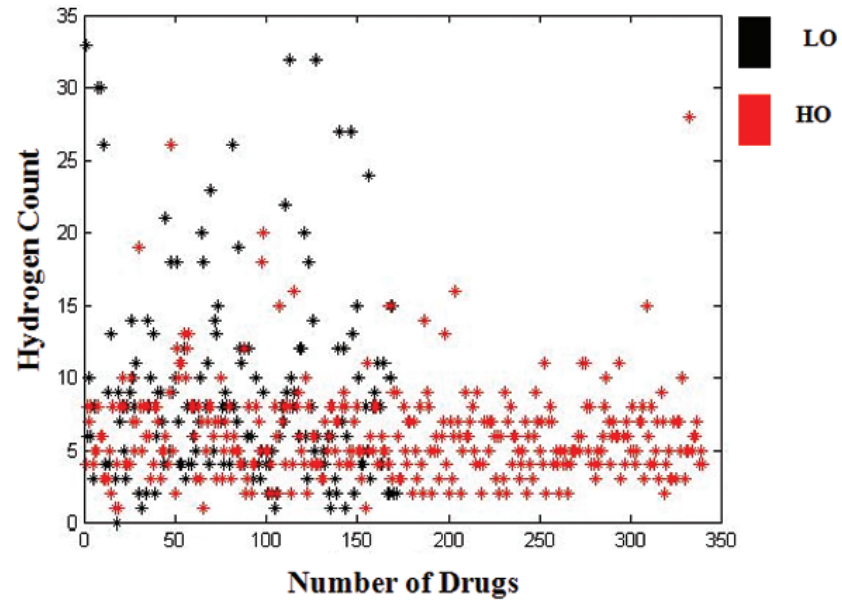

(b)

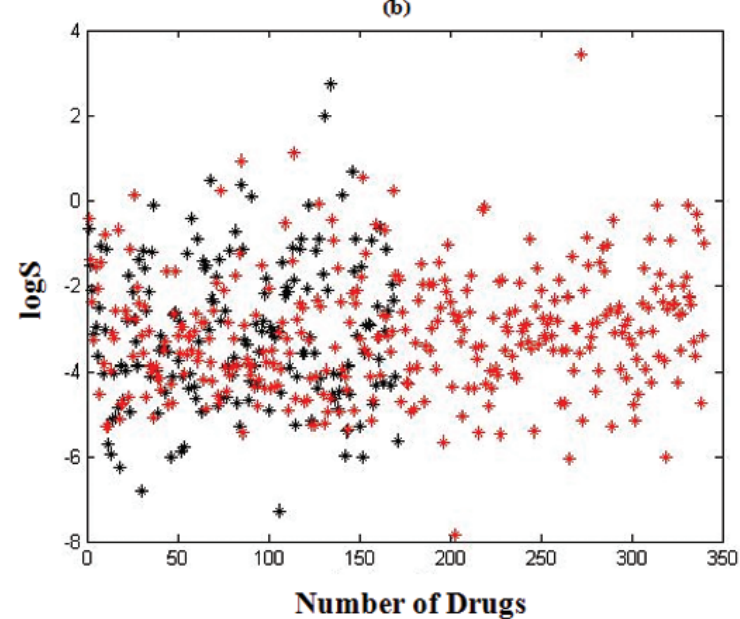

(d)

Figure 2 - Dot plot representing the distribution of descriptor values for drugs with low (LO) and high oral (HO) bioavailability. (a): Rotatable Bonds, (b): Hydrogen Count, (c): Shape Flexibility Index and (d): logS. The red and black points indicate the data of $\mathrm{LO}$ and $\mathrm{HO}$ drugs respectively.

ity of drugs (Fig. 3). Precision of SVM was found to be $4.33 \%$ and $6.67 \%$ greater than ANN and Bayesian classifier respectively. Moreover, accuracy of SVM for this dataset was found to be $2.32 \%$ and $4.66 \%$ more than ANN and Bayesian classifier respectively. Although sensitivity of ANN and SVM is same for this dataset, but RBF kernels based SVM classifier appears to be the best in terms of accuracy and precision.

\section{CONCLUSION}

The study suggests that machine learning approaches such as SVM, ANN and Bayesian classifier are efficient in binary classification based on oral bioavailability of drugs using physicochemical properties. Comparative studies of all three classifiers suggest that overall efficiency of SVM for considered dataset is better followed by ANN and Bayesian classifiers.

\section{REFERENCES}

[1] CANTU-PAZE, NEWSAM S \& KAMATH C. 2004. Feature selection in scientific applications. International Conference on Knowledge Discovery and data Mining, US.

[2] CHANG CC \& LIN CJ. 2001. LIBSVM, version 2.81, http://www.csie.ntu.edu.tw cjlin/libsvm

[3] FRANCESCO A, STEFANO L, ENZA M \& LEONARDO V. 2006. Genetic programming for Human bioavailability of drugs GECCO'6, 255-262.

[4] GOODMAN LS \& GILMAN A. 2001. The Pharmacological Basis of Therapeutics, $10^{\text {th }}$ edn. McGraw-Hill, Now York, pp. 1917-2023.

[5] HALL LM, LOWELL HH \& LEMONT BK. 2003. Modeling Drug Albumin Binding Affinity with E-State Topological Structure Representation. J. Chem. Inf. Comput. Sci., 43(6): 2120-2128. 


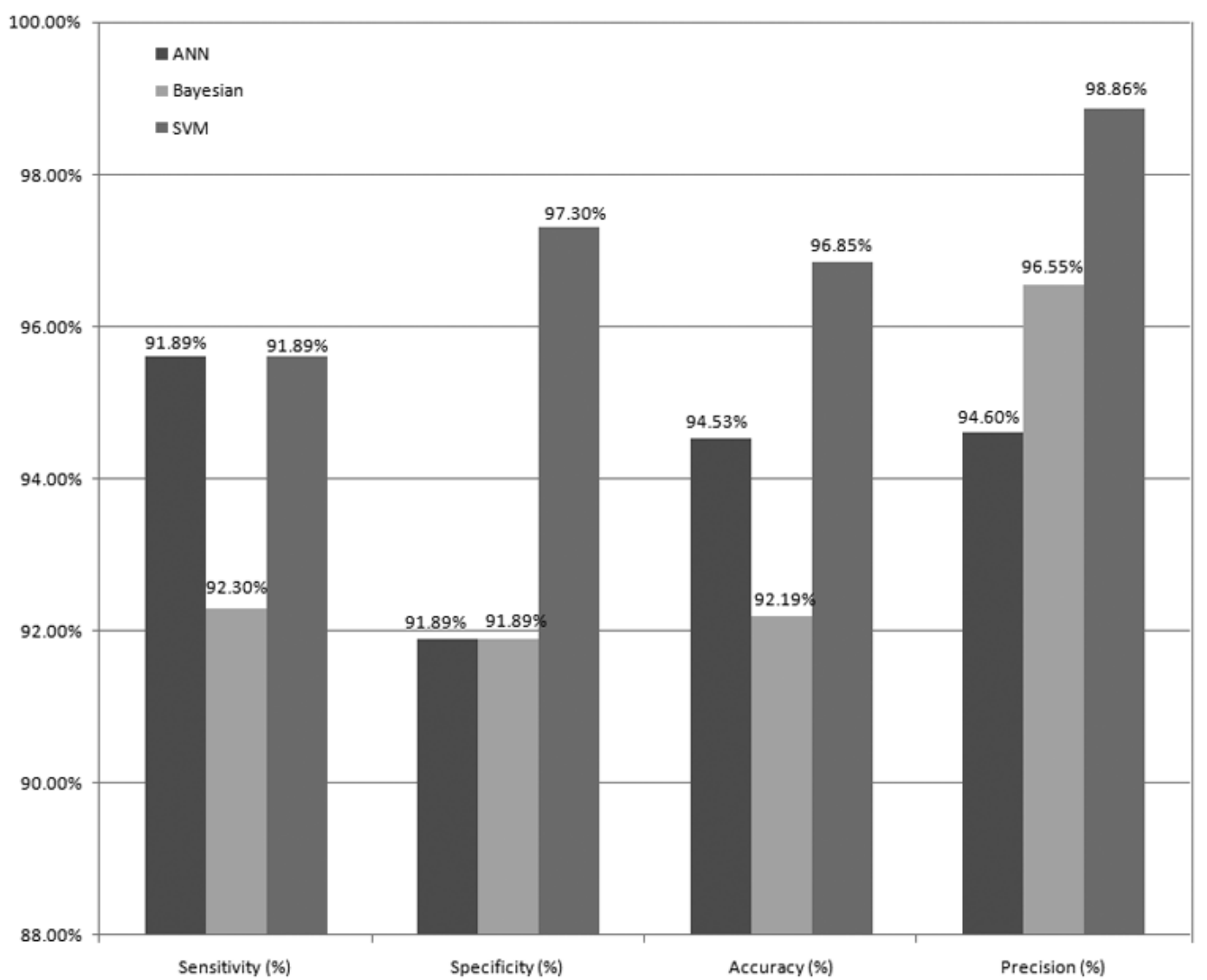

Figure 3 - Measure of efficiency of three classifiers for prediction of oral bioavailability of drugs.

[6] HOU T, WANG J, ZHANG W \& XU X. 2007. ADME evaluation in Drug Discovery: Can Oral bioavailability in humans be predicted by simple molecular property-based rules. J. Chem. Inf. Model, 47: 460-463.

[7] HSU CW, CHANG CC \& LIN CJ. 2003. A practical guide to Support Vector Classification, http://www.csie.ntu.edu.tw/ cjlin.

[8] ISABEL D, ANTONIO C, INÉS MA, ANA C, CARMEN G, MANFRED S \& ANA M. 2004. CODES/Neural Network Model: a Useful Tool for in Silico Prediction of Oral Absorption and Blood-Brain Barrier Permeability of Structurally Diverse Drugs. QSAR \& Combinatorial Science, 23(2): 89-98.

[9] JUNMEI W, GEORGE K, XIE XQ, CHENGDE W \& GEORGE $H$. 2006. Genetic Algorithm-Optimized QSPR Models for Bioavailability, Protein Binding and Urinary Excretion. J. Chem. Inf. Model, 46(6): 2674-2683.

[10] KLOPMAN G, STEFAN LR \& SAIAKHOV RD. 2002. ADME evaluation, Il: a computer model for the prediction of intestinal absorption in humans. Eur. J. Pharm. Sci., 17: 253-263.

[11] LINNANKOSKI J, MÄKELÄ JM, RANTA VP, URTTI A \& YLIPERTTULA M. 2006. Computational Prediction of Oral Drug Absorption
Based on Absorption Rate Constants in Humans. J. Med. Chem., 49(12): 3674-3681.

[12] LIPSINKI CA, LOMABARDO F, DOMINY BW \& FEENEY PJ. 1997. Experimental and computational approaches to estimate solubility and permeability in drug discovery and development settings. Adv. Drug Delivery Rev., 23: 4-25.

[13] LIU HX, HU RJ, ZHANG RS, YAO XJ, LIU MC, HU ZD \& FAN BT. 2005. The prediction of human oral absorption for diffusion ratelimited drugs based on heuristic method and support vector machine. J. Comput. Aided Mol. Des., 19: 33-46.

[14] MARTIN TK \& YVONNE A. 2009. Influence of molecular properties on oral bioavailability of lipophilic drugs - Mapping of bulkiness and different measures of polarity. Pharm. Dev. Technol., 14(3): 312-320.

[15] OPREA TI \& GOTTFRIES J. 1999. Toward Minimalistic Modeling of Oral Drug Absorption. J. Mol. Graphics Modell., 17(5): 261274.

[16] PODLOGAR BL, MUEGGE I \& BRICE LJ. 2001. Computational methods to estimate drug development parameters. Curr. Opin. Drug Disc. Dev., 4: 102-109. 
[17] SOMOGYI A, EICHELBAUM M \& GUGLER R. 2006. Prediction of bioavailability for drugs with a high first-pass effect using oral clearance data. Eur. J. of Clinical Pharm., 22(1): 85-90.

[18] TETKO IV, GASTEIGER J, TODESCHINI R, MAURI A, LIVINGSTONE D, ERTL P, PALYULIN VA, RADCHENKO EV, ZEFIROV NS, MAKARENKO AS, TANCHUK VY \& PROKOPENKO VV. 2005. Virtual computational chemistry laboratory - design and description, J. Comput. Aid. Mol. Des., 19: 453-63.

[19] TURNER JV, MADDALENA DJ \& AGATONOVIC KS. 2004. Bioavailability prediction based on molecular structure for a diverse series of drugs. Pharm. Res., 21: 68-82.

[20] USANSKY H \& SINKO PJ. 2005. Estimating human drug oral absorption kinetics from Caco-2 permeability using an absorptiondisposition model: model development and evaluation and derivation of analytical solutions for $k(a)$ and $F(a)$. J. Pharmacol. Exp. Ther., 314: 391-399.

[21] VEBER DF, JOHNSON SR, CHENG HY, SMITH BR, WARD KW \& KOPPLE KD. 2002. Molecular properties that influence the oral bioavailability of drug candidates. J. Med. Chem., 45: 2615-2623.

[22] YOSHIDA F \& TOPLISS JG. 2000. QSAR model for drug human oral bioavailability. J. Med. Chem., 43: 2575-2585.

[23] WATERBEEMD HV. 2009. Drugbioavailability, permeability, absorption, estimation of solubility and bioavailability. Bernard Testa, $2^{\text {nd }}$ edition, 40: 433-454.
[24] XUE Y, LI ZR, YAP CW, SUN LZ, CHEN X \& CHEN YZ. 2004. Effect of Molecular Descriptor Feature Selection in Support Vector Machine Classification of Pharmacokinetic and Toxicological Properties of Chemical Agents. J. Chem. Inf. Comput. Sci., 44: 1630-1638.

[25] ZHAO YH, ABRAHAM MH, LE J, HERSEY A, LUSCOMBE CN, BECK G, SHERBORNE B \& COOPER I. 2002. Rate limited steps of human oral absorption and QSAR studies. Pharma. Res., 19(10): 1446-1457.

\section{A APPENDIX}

1. Start with empty set $Y=\{\Phi\}$

2. Select the best feature $X+=\underset{X \in Y k}{\arg \max }[J(Y k+x)]$

$$
Y k=Y k+X^{+} ; k=k+1
$$

3. Select the worst feature

$$
X^{-}=\underset{X \in Y k}{\arg \max }[J(Y k-x)]
$$

4. If $J\left(Y k-X^{-}\right)>J(Y k)$ then

$Y k+1=Y k-x ; k=k+1$

go to step 3

else

go to step 2 